\title{
Non-Gaussian Halo Bias Re-examined: Mass-dependent Amplitude from the Peak-Background Split and Thresholding
}

\author{
Vincent Desjacques, ${ }^{1, *}$ Donghui Jeong, ${ }^{2, \dagger}$ and Fabian Schmidt ${ }^{2, \dagger}$ \\ ${ }^{1}$ Institute for Theoretical Physics, University of Zürich, Winterthurerstrasse 190, CH-8057 Zürich, Switzerland \\ ${ }^{2}$ California Institute of Technology, Mail Code 350-17, Pasadena, California, 91125, U.S.A.
}

(Received 20 May 2011; published 14 September 2011)

\begin{abstract}
Recent results of N-body simulations have shown that current theoretical models are not able to correctly predict the amplitude of the scale-dependent halo bias induced by primordial non-Gaussianity, for models going beyond the simplest, local quadratic case. Motivated by these discrepancies, we carefully examine three theoretical approaches based on (1) the statistics of thresholded regions, (2) a peak-background split method based on separation of scales, and (3) a peak-background split method using the conditional mass function. We first demonstrate that the statistics of thresholded regions, which is shown to be equivalent at leading order to a local bias expansion, cannot explain the mass-dependent deviation between theory and N-body simulations. In the two formulations of the peak-background split on the other hand, we identify an important, but previously overlooked, correction to the non-Gaussian bias that strongly depends on halo mass. This new term is in general significant for any primordial nonGaussianity going beyond the simplest local $f_{\mathrm{NL}}$ model. In a separate paper (to be published in PRD rapid communication), the authors compare these new theoretical predictions with $\mathrm{N}$-body simulations, showing good agreement for all simulated types of non-Gaussianity.
\end{abstract}

DOI: 10.1103/PhysRevD.84.063512

\section{INTRODUCTION}

Ongoing and future galaxy surveys will provide a large amount of data that can be exploited to constrain the physics of inflation and the very early Universe, in particular, through a measurement of the shape and amplitude of primordial non-Gaussianity (NG). Over the past few years, galaxy clustering has emerged as the most powerful largescale structure probe of primordial NG (e.g., [1-3]; for a review, see [4]). In particular, references [3,5-7] have shown that the local quadratic coupling $f_{\mathrm{NL}} \phi^{2}$ induces a scale-dependent bias

$$
\Delta \mathrm{b}_{\mathrm{I}}(k, z)=\frac{2 f_{\mathrm{NL}}\left(b_{1}^{\mathrm{E}}-1\right) \delta_{c}}{\mathcal{M}(k, z)}
$$

in the large-scale power spectrum of biased tracers. Here, $b_{1}^{\mathrm{E}}$ is the (Eulerian) linear bias factor, $\delta_{c} \approx 1.69$ is the linear critical density contrast for spherical collapse, and $\mathcal{M}(k, z) \propto D(z) k^{2} T(k)$ is the transfer function between density and the gravitational (Bardeen) potential perturbations. Numerical studies have confirmed the scaling $\Delta \mathrm{b}_{\mathrm{I}} \propto k^{-2}$ and the redshift-dependence $\Delta \mathrm{b}_{\mathrm{I}} \propto D(z)^{-1}$ [3,8-12], even though the exact amplitude of the effect remains somewhat debatable (at the $\sim 10-20 \%$ level; presumably related to the choice of halo finder [4]).

However, for other non-Gaussian models such as a local $f_{\mathrm{NL}} \phi^{2}$ model with $k$-dependent $f_{\mathrm{NL}}$, the local cubic coupling $g_{\mathrm{NL}} \phi^{3}$, or the orthogonal type, there is a much larger

\footnotetext{
*dvince@physik.uzh.ch

djeong@tapir.caltech.edu

*fabians@tapir.caltech.edu
}

PACS numbers: 98.80. $-\mathrm{k}, 98.65 .-\mathrm{r}, 98.80 . \mathrm{Cq}, 95.36 .+\mathrm{x}$

discrepancy between the analytical predictions based on the statistics of high-threshold regions [5] and the non-Gaussian bias measured from simulations [13-15]. In the $g_{\mathrm{NL}} \phi^{3}$ case, the magnitude of the non-Gaussian scale-dependent bias $\Delta \mathrm{b}_{\mathrm{I}}$ is significantly suppressed relative to the theoretical expectation on large scales $\left(k \lesssim 0.01 \mathrm{~h} \mathrm{Mpc}^{-1}\right.$ ), even for highly biased halos. The ratio of the measured to the predicted non-Gaussian bias strongly depends on the halo mass $M$ : it decreases towards low mass halos, and even reverses sign for halos with $b_{1}^{\mathrm{E}} \lesssim 2$ [13]. For the quadratic coupling with $k$-dependent $f_{\mathrm{NL}}(k) \propto k^{n_{f}}$, the discrepancy between the simulated bias and the high-peak expectation also becomes more severe as the halo mass decreases. Furthermore, the deviation depends on the sign and amplitude of the spectral index $n_{f}$ [14]. Recent numerical simulations implementing the orthogonal bispectrum shape also show systematic deviations in the measured halo bias from the high-peak expectation, in a way that the deviation becomes larger towards lower halo masses [15].

In this paper, we present a careful (re-)derivation of the effect of local and nonlocal primordial NG on the largescale clustering of tracers (such as galaxies and clusters of galaxies) using the thresholding approach [5,16,17], as well as two distinct albeit related formulations of the peak-background split (PBS). For all three approaches, we present general expressions for the non-Gaussian scale-dependent bias and apply them to models for which N-body simulations have been performed.

In the thresholding approach, we directly calculate the two-point correlation function of halos from the probability of finding a single smoothed region above some 
threshold, and the probability of finding two separate regions above the same threshold. This approach has the advantage that the thresholding process is a well-defined mathematical operation so that we can in principle calculate the correlation functions without any further approximation. We derive a general expression for the amplitude of the non-Gaussian bias in terms of the primordial $N$-point functions and the Gaussian bias parameters of the thresholded regions, without relying on the high-peak assumption usually assumed in previous studies (e.g., [5]). As the deviation between N-body simulations and the theoretical expectation is stronger for lower mass halos, such an extension of the high-peak formulation could be seen as a possible resolution. We also show that to leading order in $f_{\mathrm{NL}}, g_{\mathrm{NL}}, \ldots$, the thresholding approach is equivalent to a local bias expansion.

In the first PBS approach $[3,6,18]$, we decompose the non-Gaussian perturbations into parts that are linear, quadratic, and cubic in Gaussian fields. We separate longwavelength from short-wavelength perturbations (the two are uncorrelated for Gaussian fields, but correlated in the non-Gaussian case), and calculate the bias as the response of the halo number density to a long-wavelength density perturbation. This approach is conceptually simple and offers a clear physical picture of the impact of primordial NG on the clustering of biased tracers, by isolating the effect of the mode-coupling induced by NG. For example, for a generic primordial bispectrum, the variance of the small-scale density field is locally rescaled by longwavelength potential fluctuations. Depending on the exact shape of the bispectrum, this rescaling can be scaleindependent (local model of NG), which then leads to a scale-dependent bias as in Eq. (1); or scale-dependent (e.g., orthogonal and equilateral models), generally softening the $1 / k^{2}$-dependence. We will see that for cubicorder NG, long-wavelength perturbations not only rescale the local variance of the density field, but also induce a local skewness. Since the abundance of halos also depends on the skewness of the small-scale density field (a fact exploited when searching for NG using the mass function of e.g., galaxy clusters), this effect contributes to the nonGaussian halo bias. This first PBS approach has the advantage that it can be generically applied to any prescription for the average halo abundance (mass function). On the other hand, it assumes a clear separation between long- and short-wavelength modes, which breaks down when measuring the clustering on sufficiently small scales.

Our second PBS approach is inspired by a calculation of the scale-dependent bias factors in the Gaussian peaks model [19] (e.g., the first-order bias is $\mathrm{b}_{\mathrm{I}}(k)=$ $b_{10}+b_{01} k^{2}$. In this approach, we apply the peakbackground split directly to the non-Gaussian density field. This is done by calculating the non-Gaussian conditional mass function using an Edgeworth expansion of its Gaussian counterpart. The halo density contrast is then obtained by taking the ratio of the unconditional to conditional mass function, and expanding with respect to the large-scale density contrast. This allows us to determine the linear bias as the lowest-order coefficient in this series. This approach can in principle be applied to any excursionset mass function. As a first step, we will here formulate it under the assumption that the Press-Schechter multiplicity function describes halo abundances. On the other hand, this approach does not rely on a separation of scales. Thus, the two PBS approaches presented here make complementary assumptions.

The paper is organized as follows: we begin by reviewing the models of primordial NG considered here and spelling out our notation in Sec. II. We discuss the thresholding approach to non-Gaussian bias and point out its limitations in Sec. III. Section IV introduces the first PBS approach based on a separation of scales, while Sec. V presents the second PBS approach based on conditional mass functions. Section VI presents a comparison of the PBS and thresholding approaches. The comparison of our predictions with the results of N-body simulations is the subject of a companion Letter [20]. We conclude in Sec. VII.

\section{PRELIMINARIES}

\section{A. Four types of primordial NG}

Throughout the paper, we will apply our results to the following four models of primordial NG. We parameterize primordial NG via the $N$-point functions $(N>3)$ of the Bardeen potential $\Phi(\mathbf{x})$, a relativistic generalization of the Newtonian gravitational potential, in the matterdominated era. Note that $\Phi(\mathbf{x})$ has the opposite sign relative to the usual Newtonian gravitational potential. Since our goal is to compare analytic predictions with the outcome of N-body simulations, our set of models includes all the templates for which simulations have been performed. Our main theoretical results, however, will always be given in terms of general $\mathrm{N}$-point functions and can be straightforwardly applied to any given model of NG.

\section{Local NG}

In local primordial NG, the non-Gaussian field $\Phi$ is defined by a local Taylor expansion around a Gaussian random field $\phi$ as [21-24]

$$
\Phi(\mathbf{x})=\phi(\mathbf{x})+f_{\mathrm{NL}} \phi^{2}(\mathbf{x})+g_{\mathrm{NL}} \phi^{3}(\mathbf{x}) .
$$

Here, $f_{\mathrm{NL}}$ and $g_{\mathrm{NL}}$ are dimensionless, phenomenological parameters which we seek to constrain using cosmic microwave background (CMB) or large-scale structure (LSS) observations. This type of NG is typically produced in inflationary models with more than one scalar field. Since the primeval curvature perturbations are of magnitude $\mathcal{O}\left(10^{-5}\right)$, the cubic-order correction is negligibly small compared to the quadratic one when 
$\mathcal{O}\left(f_{\mathrm{NL}}\right) \sim \mathcal{O}\left(g_{\mathrm{NL}}\right)$. However, this condition is not satisfied by some multifield models such as the curvaton scenario, in which a large $g_{\mathrm{NL}}$ and a small $f_{\mathrm{NL}}$ can be simultaneously produced [25-29]. At leading order, the quadratic term generates a 3-point function or bispectrum,

$$
\xi_{\Phi}^{(3)}\left(\mathbf{k}_{1}, \mathbf{k}_{2}, \mathbf{k}_{3}\right)=2 f_{\mathrm{NL}}\left[P_{\phi}\left(k_{1}\right) P_{\phi}\left(k_{2}\right)+(2 \text { cyc. })\right],
$$

where (cyc.) denotes cyclic permutations of the indices, $P_{\phi}(k) \propto k^{n_{s}-4}$ is the power spectrum of the Gaussian field $\phi(\mathbf{x})$, and $n_{s}$ is its logarithmic slope. On the other hand, the cubic-order terms generate a 4-point function or trispectrum,

$\xi_{\Phi}^{(4)}\left(\mathbf{k}_{1}, \mathbf{k}_{2}, \mathbf{k}_{3}, \mathbf{k}_{4}\right)=6 g_{\mathrm{NL}}\left[P_{\phi}\left(k_{1}\right) P_{\phi}\left(k_{2}\right) P_{\phi}\left(k_{3}\right)+(3\right.$ cyc. $\left.)\right]$.

Both bispectrum and trispectrum are peaked on squeezed triangle or quadrilateral configurations, i.e., configurations where one side $\left|\mathbf{k}_{i}\right|$ is much shorter than the other sides.

\section{Scale-dependent $f_{\mathrm{NL}}$}

Next, we will consider a model in which the quadratic coupling dominates but $f_{\mathrm{NL}}$ is $k$-dependent. The primordial bispectrum takes the form [14]

$$
\begin{array}{r}
\xi_{\Phi}^{(3)}\left(\mathbf{k}_{1}, \mathbf{k}_{2}, \mathbf{k}_{3}\right)=f_{\mathrm{NL}}\left(k_{1}\right) P_{\phi}\left(k_{2}\right) P_{\phi}\left(k_{3}\right)+(5 \text { perm. }) \\
\text { with } \quad f_{\mathrm{NL}}(k)=f_{\mathrm{NL}}\left(k_{p}\right)\left(\frac{k}{k_{p}}\right)^{n_{f}},
\end{array}
$$

where $k_{p}$ is some arbitrary fixed scale, and $n_{f}$ is a spectral index.

\section{Folded and orthogonal NG}

As a third template, we will consider the folded or flattened shape, for which the primordial bispectrum reads [30]

$$
\begin{aligned}
\xi_{\Phi}^{(3)}\left(\mathbf{k}_{1}, \mathbf{k}_{2}, \mathbf{k}_{3}\right)= & 6 f_{\mathrm{NL}}\left[\left(P_{\phi}\left(k_{1}\right) P_{\phi}\left(k_{2}\right)+(2 \mathrm{cyc} .)\right)\right. \\
& +3\left(P_{\phi}\left(k_{1}\right) P_{\phi}\left(k_{2}\right) P_{\phi}\left(k_{3}\right)\right)^{2 / 3} \\
& -\left(P_{\phi}\left(k_{1}\right)^{1 / 3} P_{\phi}\left(k_{2}\right)^{2 / 3} P_{\phi}\left(k_{3}\right)\right. \\
& +(5 \text { perm. }))] .
\end{aligned}
$$

The folded shape approximates the NG due to modification of the initial Bunch-Davies vacuum in canonical singlefield inflation (the actual 3-point function is not factorizable). This template induces a scale-dependent bias on large scales with somewhat weaker $k$-dependence than the local model. [18,31]. The orthogonal template introduced by [32],

$$
\begin{aligned}
\xi_{\Phi}^{(3)}\left(\mathbf{k}_{1}, \mathbf{k}_{2}, \mathbf{k}_{3}\right)= & 6 f_{\mathrm{NL}}\left[-3\left(P_{\phi}\left(k_{1}\right) P_{\phi}\left(k_{2}\right)+(\mathrm{cyc} .)\right)\right. \\
& -8\left(P_{\phi}\left(k_{1}\right) P_{\phi}\left(k_{2}\right) P_{\phi}\left(k_{3}\right)\right)^{2 / 3} \\
& +3\left(P_{\phi}\left(k_{1}\right)^{1 / 3} P_{\phi}\left(k_{2}\right)^{2 / 3} P_{\phi}\left(k_{3}\right)\right. \\
& +(5 \text { perm. }))],
\end{aligned}
$$

gives rise to a similar non-Gaussian halo bias [18], but roughly twice as large in magnitude and opposite in sign (for fixed $f_{\mathrm{NL}}$ ) [33].

\section{Equilateral $N G$}

Finally, the equilateral type of NG, which arises in inflationary models with higher-derivative operators such as the DBI model, is well described by the factorizable form [34]

$$
\begin{aligned}
\xi_{\Phi}^{(3)}\left(\mathbf{k}_{1}, \mathbf{k}_{2}, \mathbf{k}_{3}\right)= & 6 f_{\mathrm{NL}}\left[-\left(P_{\phi}\left(k_{1}\right) P_{\phi}\left(k_{2}\right)+(\text { cyc. })\right)\right. \\
& -2\left(P_{\phi}\left(k_{1}\right) P_{\phi}\left(k_{2}\right) P_{\phi}\left(k_{3}\right)\right)^{2 / 3} \\
& +\left(P_{\phi}\left(k_{1}\right)^{1 / 3} P_{\phi}\left(k_{2}\right)^{2 / 3} P_{\phi}\left(k_{3}\right)\right. \\
& +(5 \text { perm. }))] .
\end{aligned}
$$

It can easily be verified that the signal is largest in the equilateral configurations $k_{1} \approx k_{2} \approx k_{3}$, and suppressed in the squeezed limit $k_{3} \ll k_{1} \approx k_{2}$.

\section{B. From primordial perturbations to galaxies}

In standard CDM cosmologies, galaxies form inside dark matter halos and this introduces a bias between the mass and the galaxy distributions [35]. In what follows, we shall adopt a Lagrangian picture. Namely, we express the clustering of biased tracers, such as dark matter halos of mass $M$ collapsing at redshift $z$, in terms of the statistics of the initial density perturbation $\delta_{R}(\mathbf{k}, z)$ smoothed on a scale $R$ and linearly evolved to redshift $z$, where $R$ is related to $M$ via $M=(4 \pi / 3) \bar{\rho} R^{3}$. More precisely, $\delta$ is the fractional density perturbation in synchronous gauge. Thus, the Poisson equation provides a relationship between $\delta_{R}(\mathbf{k}, z)$ and the Bardeen potential $\Phi(\mathbf{x})$ via

$$
\delta_{R}(\mathbf{k}, z)=\mathcal{M}_{R}(k, z) \Phi(\mathbf{k}),
$$

where

$$
\mathcal{M}_{R}(k, z)=\mathcal{M}(k, z) W_{R}(k)=\frac{2}{3} \frac{k^{2} T(k) g(z)}{\Omega_{m} H_{0}^{2}(1+z)} W_{R}(k) .
$$

Here, $T(k)$ is the matter transfer function normalized to unity as $k \rightarrow 0, g(z)$ is the linear growth rate of the gravitational potential normalized to unity during the matterdominated epoch, and $W_{R}(k)$ is a (spherically symmetric) window function with characteristic radius $R$. We will assume a spherical top-hat filter throughout. Note also that the matter power spectrum at redshift $z$ is related to 
the primordial curvature power spectrum through $P_{m}(k, z)=\mathcal{M}^{2}(k, z) P_{\phi}(k)$.

Regardless of the initial conditions, we shall denote the Lagrangian bias factors of dark matter halos by $b_{I}, b_{I I}, \ldots$, while Eulerian bias parameters are denoted as $b_{I}^{E}$, etc. Note that these bias parameters are generally scale-dependent. The notation $b_{1}, b_{2}, b_{1}^{\mathrm{E}}$, etc. will exclusively designate the Gaussian, scale-independent peak-background split biases. In the next section, we will also use the notation $c_{1}, c_{2}, \ldots$ for the mass-weighted, cumulative Gaussian bias parameters which appear in the thresholding approach.

We will describe the abundance of halos through their mass function $n_{h} \equiv d n / d M$ which we will assume to be of the universal form, i.e.,

$$
\bar{n}_{h}=\frac{\bar{\rho}}{M^{2}} f(\nu)\left|\frac{\partial \ln \sigma_{0 M}}{\partial \ln M}\right|,
$$

where $f(\nu)$ is the multiplicity function and $\sigma_{0 M}$ is the RMS density fluctuation on scale $M$.

Unless otherwise specified, we shall adopt in all illustrations a flat $\Lambda$ CDM cosmology with $\Omega_{m}=0.279$, $h=0.7$, and an adiabatic initial perturbations with spectral index $n_{s}=0.96$ and amplitude $A_{s}=7.96 \times 10^{-10}$ at the pivot point $k_{0}=0.02 \mathrm{Mpc}^{-1}$ (corresponding to a normalization $\sigma_{8} \approx 0.81$ ). These values are consistent with the latest CMB constraints from WMAP7 [36].

\section{STATISTICS OF THRESHOLDED REGIONS}

In this section, we shall present the derivation of the scale-dependent non-Gaussian bias using the statistics of regions above threshold [16,17], without invoking the high-threshold (high-peak) approximation. Several concepts and results introduced in this section will be employed later in the paper.

\section{A. Probability densities}

In the Press-Schechter approach [37], virialized objects are identified with high-density regions in the linear density field. The two-point correlation function of thresholded regions, $\xi_{>\nu}(r)$, can be calculated once the probability $P_{1}$ of finding a region whose overdensity is above the threshold $\delta_{c} \approx 1.69$ [38], and the probability $P_{2}$ of finding two such regions separated by a distance $r \equiv\left|\mathbf{x}_{2}-\mathbf{x}_{1}\right|$, are known. It is convenient to express the results in terms of the significance (peak height) $\nu \equiv \delta_{c} / \sigma_{0 s}$, where $\sigma_{0 s}$ is the r.m.s. variance of the density field smoothed on scale $R_{s}$. The correlation function is then given by [35]:

$$
\xi_{>\nu}(r)=\frac{P_{2}(>\nu, r)}{\left[P_{1}(>\nu)\right]^{2}}-1
$$

$\xi_{>\nu}(r)$ is commonly interpreted as describing the 2-point correlation of halos above mass $M$ corresponding to the smoothing length $R_{s}$. For any non-Gaussian initial density field, $P_{1}$ and $P_{2}$ can be expressed in terms of the $N$-point connected correlation functions as follows [39]:

$$
\begin{aligned}
& P_{1}(>\nu)=\frac{1}{\sqrt{2 \pi}} \int_{\nu}^{\infty} d y \exp \left[\sum_{N=3}^{\infty}(-1)^{N} \frac{w_{s}^{(N, 0)}}{N !} \frac{d^{N}}{d y^{N}}\right] e^{-y^{2} / 2} \\
& P_{2}(>\nu, r)= \frac{1}{2 \pi} \int_{\nu}^{\infty} d y_{1} \int_{\nu}^{\infty} d y_{2} \exp \left[\sum_{N=2}^{\infty} \sum_{m=0}^{N}(-1)^{N}\right. \\
&\left.\times \frac{w_{s}^{(N, m)}(r)}{m !(N-m) !} \frac{\partial^{N}}{\partial y_{1}^{m} \partial y_{2}^{N-m}}\right] e^{-(1 / 2)\left(y_{1}^{2}+y_{2}^{2}\right)} .
\end{aligned}
$$

For shorthand convenience, we will hereafter omit the explicit $z$-dependence of $\delta_{s}(\mathbf{x}) \equiv \delta_{R_{s}}(\mathbf{x})$ and $\mathcal{M}_{s}(k) \equiv \mathcal{M}_{R_{s}}(k)$. We have also defined

$$
w_{s}^{(N, m)}(r) \equiv \begin{cases}w_{s}^{(2, m)}=\xi_{s}^{(2, m)}(r) / \sigma_{0 s}^{2} & (m=1) \\ w_{s}^{(2, m)}=0 & (m=1 \text { or } 2), \\ w_{s}^{(N, m)}=\xi_{s}^{(N, m)}(r) / \sigma_{0 s}^{N} & (N>2)\end{cases}
$$

where

$$
\xi_{s}^{(N, m)}(r) \equiv\langle\underbrace{\delta_{s}\left(\mathbf{x}_{1}\right) \cdots \delta_{s}\left(\mathbf{x}_{1}\right)}_{m \text { times }} \underbrace{\delta_{s}\left(\mathbf{x}_{2}\right) \cdots \delta_{s}\left(\mathbf{x}_{2}\right)}_{N-m \text { times }}\rangle_{c}
$$

is the $N$-point connected correlation function evaluated at two different locations $\mathbf{x}_{1}$ and $\mathbf{x}_{2}$. Note that the correlation $w_{s}^{(N, 0)}=w_{s}^{(N, N)}$ is evaluated at zero lag, and that the probability densities $P_{1}$ and $P_{2}$ depend explicitly on the smoothing scale $R_{s}$ through the functions $w_{s}^{(N, m)}$ and the peak height $\nu \equiv \delta_{c} / \sigma_{0 s}$.

\section{B. Bias parameters for a Gaussian density field}

It is instructive to first perform the calculation for Gaussian initial conditions. Later, we shall use the Gaussian bias derived in this Section to identify the coefficients of the non-Gaussian scale-dependent bias.

\section{Gaussian bias factors from a peak-background split}

When the underlying smoothed density field obeys the Gaussian statistics, the probability $P_{1}$ of exceeding the threshold $\nu$ is given by

$$
P_{1}(>\nu)=\frac{1}{\sqrt{2 \pi}} \int_{\nu}^{\infty} d x e^{-x^{2} / 2}=\frac{1}{2} \operatorname{erfc}\left(\frac{\nu}{\sqrt{2}}\right) .
$$

In the peak-background split approach, one considers the effect of adding a long-wavelength (background) perturbation $\delta_{l}$ of characteristic wavelength $R_{l} \gg R_{s}$ to the small-scale density field (peak) $\delta_{s}$. Assuming that $\delta_{l}$ is independent of $\delta_{s}$, it is clear that adding $\delta_{l}$ is equivalent to reducing the threshold $\nu \rightarrow\left(\delta_{c}-\delta_{l}\right) / \sigma_{0 s}$; thus, $P_{1}\left(>\nu, \delta_{l}\right)$, the probability $P_{1}$ in the large-scale overdensity $\delta_{l}$ is given by 


$$
P_{1}\left(>\nu, \delta_{l}\right)=P_{1}\left(>\nu-\frac{\delta_{l}}{\sigma_{0 s}}\right) .
$$

We define the peak-background split bias factors $c_{N}$ as the fractional change of $P_{1}$ with $\delta_{l}$ via

$$
c_{N} \equiv \frac{1}{P_{1}(>\nu)} \frac{d^{N} P_{1}\left(>\nu, \delta_{l}\right)}{d \delta_{l}^{N}},
$$

so that

$$
\begin{aligned}
c_{N}(\nu) & =\left(-\frac{1}{\sigma_{0 s}}\right)^{N} \frac{1}{P_{1}(>\nu)} \frac{d^{N}\left[P_{1}(>\nu)\right]}{d \nu^{N}} \\
& =\sqrt{\frac{2}{\pi}}\left[\operatorname{erfc}\left(\frac{\nu}{\sqrt{2}}\right)\right]^{-1} \frac{e^{-\nu^{2} / 2}}{\sigma_{0 s}^{N}} H_{N-1}(\nu) .
\end{aligned}
$$

Here, $H_{N}$ is the Hermite polynomial defined by

$$
H_{N}(x) \equiv(-1)^{N} e^{x^{2} / 2} \frac{d^{N}}{d x^{N}}\left(e^{-x^{2} / 2}\right) .
$$

Note that we adopt the so-called probabilists' convention for the Hermite polynomials. It is related to the so-called physicists' convention by

$$
H_{N}^{\text {phys }}(x)=2^{N / 2} H_{N}(\sqrt{2} x) .
$$

Explicit expressions for the first five Hermite polynomials are

$$
\begin{aligned}
& H_{0}(x)=1, \\
& H_{1}(x)=x, \\
& H_{2}(x)=x^{2}-1, \\
& H_{3}(x)=x^{3}-3 x, \\
& H_{4}(x)=x^{4}-6 x^{2}+3 .
\end{aligned}
$$

Since $H_{N} \rightarrow \nu^{N}$ for large $\nu$, we see that in the high-peak limit $(\nu \gg 1)$,

$$
c_{N} \approx \nu H_{N-1}(\nu) / \sigma_{0 s}^{N} \approx \nu^{N} / \sigma_{0 s}^{N} .
$$

The one-point probability Eq. (17) and bias parameters Eq. (20) are cumulative. They describe the number density and bias of all peaks above the threshold $\nu$ at fixed smoothing scale $R_{s}$. To relate these quantities to the mass function and bias of dark matter halos, we follow Press \& Schechter [37] and interpret $P_{1}$ as the fraction of the Lagrangian volume occupied by halos of mass exceeding $M$. Therefore, the halo number density follows upon dividing the derivative of $P_{1}$ w.r.t. mass by $M / \bar{\rho}$,

$$
\begin{aligned}
\bar{n}_{h}(M) & =-2 \frac{\bar{\rho}}{M} \frac{d}{d M} P_{1}(>\nu) \\
& =2 \frac{\bar{\rho}}{M^{2}} \frac{\nu e^{-\nu^{2} / 2}}{\sqrt{2 \pi}}\left|\frac{d \ln \sigma_{0 s}}{d \ln M}\right|,
\end{aligned}
$$

where the factor of 2 is introduced to account for the fact that regions with $\delta<\delta_{c}$ may be embedded in regions with $\delta>\delta_{c}$ on scale $>R_{s}$ (clouds-in-clouds). Thus, Eq. (25) is of the form Eq. (11) with $f(\nu)=\sqrt{2 / \pi} \nu \exp \left(-\nu^{2} / 2\right)$. Conversely, integrating Eq. (25) yields

$$
P_{1}(>\nu)=\frac{1}{2 \bar{\rho}} \int_{M}^{\infty} d M^{\prime} M^{\prime} \bar{n}_{h}\left(M^{\prime}\right) .
$$

Inserting this into Eq. (16), we find that the $c_{N}$ are massweighted cumulative bias factors,

$$
c_{N}=\left[\int_{M}^{\infty} d M^{\prime} M^{\prime} \bar{n}_{h}\left(M^{\prime}\right)\right]^{-1} \int_{M}^{\infty} d M^{\prime} M^{\prime} \bar{n}_{h}\left(M^{\prime}\right) b_{N}\left(M^{\prime}\right),
$$

where

$$
b_{N}(M)=\frac{1}{\nu_{M}} \frac{H_{N+1}\left(\nu_{M}\right)}{\sigma_{0 M}^{N}}
$$

are the peak-background split biases derived from the Press-Schechter mass function. Here, $\nu_{M}$ and $\sigma_{0 M}$ denote the significance and r.m.s. density fluctuation on the mass scale $M$. It is only in the high-peak limit $(\nu \gg 1)$ that the mass-weighted cumulative bias $c_{N}$ and the bias $b_{N}(M)$ asymptote to the same values [Eq. (24)].

So far, we have not yet specified any prescription for how to go from the bias parameter $c_{N}$ to the clustering of tracers. This will be elucidated in the next section, where we calculate the correlation function of thresholded regions directly.

\section{Gaussian bias factors from the correlation of thresholded regions}

In this section, we present the calculation of the twopoint correlation function $\xi_{>\nu}(r)$ of thresholded regions assuming Gaussian initial conditions, and show that the cumulative mass-weighted biases $c_{N}$ obtained with the peak-background split coincide with the bias parameters arising in $\xi_{>\nu}(r)$.

Observing that, for Gaussian initial conditions, all the connected correlation functions $\xi_{s}^{(N, m)}$ with $N>2$ vanish, we can express $\xi_{>\nu}(r)$ as

$$
\begin{aligned}
\xi_{>\nu}(r) \equiv & \frac{P_{2}(>\nu, r)}{\left[P_{1}(>\nu)\right]^{2}}-1 \\
= & \frac{2}{\pi}\left[\operatorname{erfc}\left(\frac{\nu}{\sqrt{2}}\right)\right]^{-2} \int_{\nu}^{\infty} d y_{1} \int_{\nu}^{\infty} d y_{2} \\
& \times \exp \left[\frac{\xi_{s}(r)}{\sigma_{0 s}^{2}} \frac{\partial^{2}}{\partial y_{1} \partial y_{2}}\right] e^{-(1 / 2)\left(y_{1}^{2}+y_{2}^{2}\right)}-1 .
\end{aligned}
$$

Here, $\xi_{s}(r) \equiv \xi_{s}^{(2,1)}$ is the 2-point density correlation smoothed on scale $R_{S}$. On employing the definition of $H_{N}(x)$, we can further simplify the double integration as 


$$
\begin{aligned}
& \int_{\nu}^{\infty} d y_{1} \int_{\nu}^{\infty} d y_{2} \exp \left[\frac{\xi_{s}(r)}{\sigma_{0 s}^{2}} \frac{\partial^{2}}{\partial y_{1} \partial y_{2}}\right] e^{-(1 / 2)\left(y_{1}^{2}+y_{2}^{2}\right)} \\
& =\frac{\pi}{2}\left[\operatorname{erfc}\left(\frac{\nu}{\sqrt{2}}\right)\right]^{2}+\sum_{N=1}^{\infty} \frac{\left[\xi_{s}(r)\right]^{N}}{N ! \sigma_{0 s}^{2 N}}\left[H_{N-1}(\nu)\right]^{2} e^{-\nu^{2} / 2}
\end{aligned}
$$

Therefore, we find that the 2-point correlation function of thresholded regions is given by [41]

$$
\xi_{>\nu}(r)=\frac{2}{\pi}\left[\operatorname{erfc}\left(\frac{\nu}{\sqrt{2}}\right)\right]^{-2} \sum_{N=1}^{\infty} \frac{\left[\xi_{s}(r)\right]^{N}}{N ! \sigma_{0 s}^{2 N}}\left[H_{N-1}(\nu)\right]^{2} e^{-\nu^{2} / 2}
$$

Next, on substituting the expression of the cumulative peak-background split bias factors Eq. (17), we can recast the peak correlation function into the series

$$
\xi_{>\nu}(r)=\sum_{N=1}^{\infty} \frac{c_{N}^{2}}{N !}\left[\xi_{s}(r)\right]^{N} .
$$

If we compare the expression for $\xi_{>\nu}(r)$ to that obtained from a local bias expansion [42] of the density $\delta_{>\nu}$ of regions above threshold,

$$
\delta_{>\nu}(\boldsymbol{x})=\sum_{N=1}^{\infty} \frac{\tilde{c}_{N}}{N !}\left[\delta_{s}(\boldsymbol{x})\right]^{N},
$$

we see that the coefficient $\tilde{c}_{N}$ is different from the $c_{N}$ appearing in the correlation function: when calculating $\xi_{>\nu}=\left\langle\delta_{>\nu}\left(\mathbf{x}_{1}\right) \delta_{\nu}\left(>\mathbf{x}_{2}\right)\right\rangle$, the coefficient of $\left[\xi_{s}(r)\right]^{N}$ includes not only $\tilde{c}_{N}^{2}$, but also terms such as $\tilde{c}_{N} \tilde{c}_{N+2 m} \sigma_{0 s}^{2 m}$ for all positive integers $m \leq N / 2$. This clearly shows that the bias parameters $c_{N}$ from the peak-background split are to be seen as "renormalized" bias parameters [43] which take all the higher-order moments into account, and thus truly are the coefficients of the observed correlation function of (in this case) thresholded regions.

\section{Two-point correlation function of thresholded regions with $\mathrm{NG}$}

In the presence of primordial NG, all the correlation functions $\xi_{s}^{(N, m)}$ are in principle necessary to determine $P_{1}(>\nu)$ and $P_{2}(>\nu, r)$. Here, we will restrict ourselves to the leading-order corrections linear in the correlations $\xi_{s}^{(N, m)}$. We derive a general expression for the scaledependent non-Gaussian bias induced by a primordial $N$-point function $\xi_{\Phi}^{(N)}$.

\section{Relation to local deterministic bias}

First, we show that the leading order contribution to the two-point correlation function of thresholded regions, which includes terms linear in the connected correlations functions $\xi_{s}^{(N, m)}$ only, is consistent with the result from a local deterministic bias ansatz. Linearizing the exponential factors in Eqs. (13) and (14), we obtain

$$
\begin{gathered}
P_{1}(>\nu) \approx \frac{1}{2} \operatorname{erfc}\left(\frac{\nu}{\sqrt{2}}\right)+\sum_{N=3}^{\infty} \frac{1}{\sqrt{2 \pi}} \frac{w_{s}^{(N, 0)}}{N !} H_{N-1}(\nu) e^{-\nu^{2} / 2} \\
P_{2}(>\nu, r) \approx\left[\frac{1}{2} \operatorname{erfc}\left(\frac{\nu}{\sqrt{2}}\right)\right]^{2}+\sqrt{\frac{1}{2 \pi}} \operatorname{erfc}\left(\frac{\nu}{\sqrt{2}}\right) \sum_{N=3}^{\infty} \frac{w_{s}^{(N, 0)}}{N !} H_{N-1}(\nu) e^{-\nu^{2} / 2} \\
+\frac{1}{2 \pi} \sum_{N=2}^{\infty} \sum_{m=1}^{N-1} \frac{w_{s}^{(N, m)}}{m !(N-m) !} H_{m-1}(\nu) H_{N-m-1}(\nu) e^{-\nu^{2}},
\end{gathered}
$$

where we have neglected terms beyond linear order. Thus, the two-point correlation function of thresholded regions reads

$$
\xi_{>\nu}(r)=\frac{2}{\pi}\left[\operatorname{erfc}\left(\frac{\nu}{\sqrt{2}}\right)\right]^{-2} \sum_{N=2}^{\infty} \sum_{m=1}^{N-1} \frac{w_{s}^{(N, m)}}{m !(N-m) !} H_{m-1}(\nu) H_{N-m-1}(\nu) e^{-\nu^{2}}=\sum_{N=2}^{\infty} \sum_{m=1}^{N-1} \frac{c_{m} c_{N-m}}{m !(N-m) !} \xi_{s}^{(N, m)}(r) .
$$

As can easily be seen, a local deterministic mapping

$$
\delta_{>\nu}(\boldsymbol{x})=\sum_{N=0}^{\infty} \frac{c_{N}}{N !}\left[\delta_{s}(\boldsymbol{x})\right]^{N},
$$

yields the same result at leading order (the renormalization of the bias parameters $c_{N}$ discussed in the previous section for the Gaussian case will apply at second and higherorder). This shows that, at first order in $\xi_{s}^{(N, m)}$, the correlation of thresholded regions with primordial $\mathrm{NG}$ is equivalent to a local deterministic bias relation. Note that, for non-Gaussian initial conditions, an effective first-order bias defined through $c_{1, \text { eff }} \equiv \sqrt{\xi_{>\nu}(r) / \xi_{s}(r)}$ is generally scale-dependent. 


\section{Power spectrum of thresholded regions}

We now Fourier-transform Eq. (30), and investigate the separate terms. For simplicity and without loss of generality, we will assume that a single non-Gaussian $N$-point function $(N>3)$ dominates. We then have

$$
P_{>\nu}(k)=c_{1}^{2} P_{s}(k)+\sum_{m=1}^{N-1} \frac{c_{m} c_{N-m}}{m !(N-m) !} \tilde{\xi}_{s}^{(N, m)}(k) .
$$

Here, $P_{s}(k)=W_{R_{s}}^{2}(k) P(k)$ is the matter power spectrum smoothed on scale $R_{s}$. Let us consider the term $m=1$ first. It is in fact identical to the term $m=N-1$. We have

$$
\begin{aligned}
\tilde{\xi}_{s}^{(N, 1)}(k)= & \prod_{i=1}^{N-1}\left(\int \frac{d^{3} k_{i}}{(2 \pi)^{3}}\right) \xi_{s}^{(N)}\left(\mathbf{k}, \mathbf{k}_{1}, \ldots, \mathbf{k}_{N-1}\right) \\
& \times(2 \pi)^{3} \delta_{D}\left(\mathbf{k}+\mathbf{k}_{1}+\ldots+\mathbf{k}_{N-1}\right) \\
= & \mathcal{M}_{s}(k) \prod_{i=1}^{N-2}\left(\int \frac{d^{3} k_{i}}{(2 \pi)^{3}} \mathcal{M}_{s}\left(k_{i}\right)\right) \mathcal{M}_{s}(q) \\
& \times \xi_{\Phi}^{(N)}\left(\mathbf{k}, \mathbf{k}_{1}, \ldots, \mathbf{k}_{N-2}, \boldsymbol{q} ; X\right) .
\end{aligned}
$$

Here, $\boldsymbol{q}=-\mathbf{k}_{1}-\ldots-\mathbf{k}_{N-2}-\mathbf{k}$ and $X$ are a set of variables characterizing the primordial $N$-point function such as $f_{\mathrm{NL}}, g_{\mathrm{NL}}, n_{f}$, depending on the details of the model of NG. In the second line, we have used the fact that the matter $N$-point function is related to the $N$-point function of the potential $\Phi$ through

$$
\xi_{s}^{(N)}\left(\boldsymbol{k}_{1}, \ldots, \boldsymbol{k}_{N}\right)=\left(\prod_{i=1}^{N} \mathcal{M}_{s}\left(k_{i}\right)\right) \xi_{\Phi}^{(N)}\left(\mathbf{k}_{1}, \ldots, \mathbf{k}_{N} ; X\right) .
$$

Note that the scaling of $\tilde{\xi}_{s}^{(N, 1)}$ in the large-scale limit $(k \rightarrow 0)$ depends on the scaling of $\xi_{\Phi}^{(N)}$ in the squeezed limit, where one argument $(k)$ is much smaller than the others $\left(k_{1}, \ldots, k_{N-2}, q\right)$.

Next, consider the term with $m=2$ (again, it is equal to the term $m=N-2$ ). A similar calculation leads to

$$
\begin{aligned}
\tilde{\xi}_{s}^{(N, 2)}(k)= & \prod_{i=1}^{N-1}\left(\int \frac{d^{3} k_{i}}{(2 \pi)^{3}}\right) \xi_{s}^{(N)}\left(\mathbf{k}-\mathbf{k}_{1}, \mathbf{k}_{1}, \ldots, \mathbf{k}_{N-1}\right) \\
& \times(2 \pi)^{3} \delta_{D}\left(\mathbf{k}+\mathbf{k}_{2}+\ldots+\mathbf{k}_{N-1}\right) \\
= & \prod_{i=1}^{N-2}\left(\int \frac{d^{3} k_{i}}{(2 \pi)^{3}} \mathcal{M}_{s}\left(k_{i}\right)\right) \mathcal{M}_{s}\left(\left|\mathbf{k}-\mathbf{k}_{1}\right|\right) \mathcal{M}_{s}(q) \\
& \times \xi_{\Phi}^{(N)}\left(\mathbf{k}-\mathbf{k}_{1}, \mathbf{k}_{1}, \ldots, \mathbf{k}_{N-2}, \boldsymbol{q} ; X\right)
\end{aligned}
$$

where now $\boldsymbol{q}=-\mathbf{k}-\mathbf{k}_{2}-\ldots-\mathbf{k}_{N-2}$. In the large-scale (small-k) limit, $\left|\mathbf{k}-\mathbf{k}_{1}\right| \gg k$, so that $\tilde{\xi}_{s}^{(N, 2)}$ approaches a constant. One can easily verify that this also holds for all $m \geq 3$ terms. On large scales, these terms thus all add white-noise contributions to the power spectrum of thresholded regions, and only the terms with $m=1, N-1$ contribute to the scale-dependent bias. Note, however, that the white-noise corrections which appear for NG of order $N \geq 4$ can be quite significant [13].

A general feature of the non-Gaussian power spectrum of tracers in the thresholding approach is now clear: the presence of a primordial $N$-point function generates a dependence of $P_{>\nu}(k)$ on the bias $c_{N-1}$ through $\tilde{\xi}_{s}^{(N, 1)}(k)$, which depends on the scaling of $\xi_{\Phi}^{(N)}$ in the squeezed limit. The former has also been pointed out by [44-46], who studied the non-Gaussian bias in the local, constant- $f_{\mathrm{NL}}$ model. As shown here, this conclusion also holds if we were to consider any local bias mapping of the form Eq. (36) (at leading order in the non-Gaussian $N$-point function). We can then rewrite Eq. (37) as

$$
\begin{aligned}
P_{>\nu}(k)= & c_{1}^{2} P_{s}(k)+2 \frac{c_{1} c_{N-1}}{(N-1) !} \tilde{\xi}_{s}^{(N, 1)}(k) \\
= & {\left[c_{1}^{2}+2 \frac{4}{(N-1) !} c_{1} c_{N-1} \sigma_{0 s}^{2}\right.} \\
& \left.\times \mathcal{M}_{s}^{-1}(k) \mathcal{F}_{s}^{(N)}(k, X)\right] P_{s}(k) .
\end{aligned}
$$

The factor of 2 comes from the sum of the $m=1, N-1$ terms, and we have introduced the shape factor

$$
\begin{aligned}
\mathcal{F}_{R}^{(N)}(k, X) \equiv & \frac{\mathcal{M}_{R}^{-1}(k)}{4 \sigma_{0 R}^{2} P_{\phi}(k)} \tilde{\xi}_{R}^{(N, 1)}(k) \\
= & \frac{1}{4 \sigma_{0 R}^{2} P_{\phi}(k)}\left\{\prod_{i=1}^{N-2} \int \frac{d^{3} k_{i}}{(2 \pi)^{3}} \mathcal{M}_{R}\left(k_{i}\right)\right\} \\
& \times \mathcal{M}_{R}(q) \xi_{\Phi}^{(N)}\left(\boldsymbol{k}_{1}, \cdots, \boldsymbol{k}_{N-2}, \boldsymbol{q}, k \hat{\mathbf{z}} ; X\right),
\end{aligned}
$$

where $\hat{\mathbf{z}}$ is some arbitrary unit vector. Noting that $P_{>\nu}=$ $\left(c_{1}^{2}+2 c_{1} \Delta c_{1}\right) P_{s}$ to leading order in the non-Gaussian corrections, we can identify the scale-dependent correction to the linear bias as

$$
\Delta c_{1}(k)=\frac{4 c_{N-1}}{(N-1) !} \sigma_{0 s}^{2} \frac{\mathcal{F}_{s}^{(N)}(k)}{\mathcal{M}_{s}(k)} .
$$

In the rest of this section, we derive the non-Gaussian correction to the clustering of thresholded regions for the four models of primordial NG we consider in this paper. It will prove useful to define general spectral moments through

$$
\sigma_{\alpha R}^{2} \equiv \frac{1}{2 \pi^{2}} \int_{0}^{\infty} d k k^{2(\alpha+1)} P_{\phi}(k) \mathcal{M}_{R}^{2}(k)
$$

\section{Local NG}

For the cubic local model, described by primordial three- and four-point functions [Sec. II A 1], Eq. (41) becomes 


$$
\begin{aligned}
P_{>\nu}(k)= & {\left[c_{1}^{2}+4 c_{1} c_{2} \sigma_{0 s}^{2} \mathcal{M}_{s}^{-1}(k) \mathcal{F}_{s}^{(3)}(k, X)\right.} \\
& \left.+\frac{4 c_{1} c_{3}}{3} \sigma_{0 s}^{2} \mathcal{M}_{s}^{-1}(k) \mathcal{F}_{s}^{(4)}(k, X)\right] P_{s}(k),
\end{aligned}
$$

where $\mathcal{F}_{s}^{(3)}\left(k, f_{\mathrm{NL}}\right)$ is precisely equal to the form factor introduced by $[5,31]$. Focusing on the quadratic case first, note that on large scales, $\mathcal{F}_{s}^{(3)} \simeq f_{\mathrm{NL}}$ and the power spectrum for $g_{\mathrm{NL}}=0$ becomes

$$
P_{>\nu}(k)=\left[c_{1}^{2}+4 f_{\mathrm{NL}} c_{1} c_{2} \sigma_{0 s}^{2} \mathcal{M}_{s}^{-1}(k)\right] P_{s}(k)
$$

From the above equation, it is clear that the scaledependence of the non-Gaussian bias is $\Delta b(k) \propto$ $\mathcal{M}_{s}^{-1}(k) \propto k^{-2}$. For high thresholds, $\nu \gg 1$ in particular, the Gaussian bias parameters $c_{N}$ approach $\nu^{N} / \sigma_{0 s}^{N}$ so that we can approximate the coefficient of the non-Gaussian correction as $c_{2} \sigma_{0 s}^{2} \simeq c_{1} \delta_{c}$. Therefore, we recover the expression of [5],

$$
P_{>\nu}(k) \stackrel{\nu \gg 1}{=} b_{1}^{2}\left[1+4 f_{\mathrm{NL}} \frac{\delta_{c}}{\mathcal{M}_{s}(k)}\right] P_{s}(k),
$$

upon replacing $c_{1}$ with $b_{1}$ (i.e., assuming a narrow mass bin).

For the local $g_{\mathrm{NL}} \phi^{3}$ model, note that

$$
P_{s}(k)=\mathcal{M}_{s}^{2}(k)\left[1+6 g_{\mathrm{NL}} \sigma_{\phi}^{2}\right] P_{\phi}(k)
$$

The matter power spectrum $P_{s}(k)$ thus contains $\sigma_{\phi}^{2} \equiv\left\langle\phi^{2}\right\rangle$, which has a logarithmic divergence for both large and small scales [47]. In reality, the finite survey size and the free-streaming scale of dark matter provide low- and high- $k$ cut-offs. In simulations, the finite box size and the resolution provide such cutoffs [13].

On large scales, the shape factor $\mathcal{F}_{s}^{(4)}$ generated by the local trispectrum rapidly converges towards $(3 / 4) g_{\mathrm{NL}} \sigma_{0 s}^{2} S_{s, \text { loc }}^{(3)}$, where

$$
\begin{aligned}
S_{s, \text { loc }}^{(3)} \equiv & \frac{6}{\sigma_{0 s}^{4}} \int \frac{d^{3} k_{1}}{(2 \pi)^{3}} \mathcal{M}_{s}\left(k_{1}\right) P_{\phi}\left(k_{1}\right) \\
& \times \int \frac{d^{3} k_{2}}{(2 \pi)^{3}} \mathcal{M}_{s}\left(k_{2}\right) P_{\phi}\left(k_{2}\right) \mathcal{M}_{s}\left(\left|\boldsymbol{k}_{1}+\boldsymbol{k}_{2}\right|\right),
\end{aligned}
$$

is the skewness parameter of the density field smoothed on scale $R_{s},\left\langle\delta_{s}^{3}\right\rangle /\left\langle\delta_{s}^{2}\right\rangle^{2}$, in a local $f_{\mathrm{NL}}$ model with $f_{\mathrm{NL}}=1$. Therefore, the non-Gaussian contribution to the power spectrum in a pure $g_{\mathrm{NL}}$ model becomes

$$
\Delta P_{>\nu}(k) \stackrel{k \rightarrow 0}{=} c_{1} c_{3} g_{\mathrm{NL}} \sigma_{0 s}^{4} S_{s, \mathrm{loc}}^{(3)} \mathcal{M}_{s}(k) P_{\phi}(k) .
$$

Note that the non-Gaussian bias also has a scale-dependence of $k^{-2}$. For high peaks $\nu \gg 1, c_{1} c_{3} \sigma_{0 s}^{4}=c_{1}^{2} \delta_{c}^{2}$, and we recover Eq. (21) of [13] upon replacing $c_{1}$ by $b_{1}$. In general however, the correct coefficient in the thresholding calculation is the third-order bias $c_{3}$.

\section{Scale-dependent and nonlocal NG}

For the $k$-dependent local bispectrum Eq. (5), the power spectrum of thresholded regions is

$$
P_{>\nu}(k)=\left[c_{1}^{2}+4 c_{1} c_{2} \sigma_{0 s}^{2} \mathcal{M}_{s}^{-1}(k) \mathcal{F}_{s}^{(3)}\left(k, f_{\mathrm{NL}}, n_{f}\right)\right] P_{s}(k),
$$

where the redshift independent function $\mathcal{F}_{s}^{(3)}\left(k, f_{\mathrm{NL}}, n_{f}\right)$ is computed from Eq. (42) on inserting Eq. (5):

$$
\begin{aligned}
\mathcal{F}_{s}^{(3)}\left(k, f_{\mathrm{NL}}, n_{f}\right)= & \frac{1}{2 \sigma_{0 s}^{2}} \frac{f_{\mathrm{NL}}\left(k_{p}\right)}{k_{p}^{n_{f}}} \int \frac{d^{3} q}{(2 \pi)^{3}} \mathcal{M}_{s}(q) \\
& \times \mathcal{M}_{s}(|\mathbf{k}-\mathbf{q}|) P_{\phi}(q) \\
& \times\left[k^{n_{f}} \frac{P_{\phi}(|\mathbf{k}-\mathbf{q}|)}{P_{\phi}(k)}+2 q^{n_{f}}\right]
\end{aligned}
$$

On large scales, the shape factor converges towards

$$
\begin{aligned}
\mathcal{F}_{s}^{(3)}\left(k, f_{\mathrm{NL}}, n_{f}\right) & \stackrel{k \rightarrow 0}{=} \frac{1}{\sigma_{0 s}^{2}} \frac{f_{\mathrm{NL}}\left(k_{p}\right)}{k_{p}^{n_{f}}} \int \frac{d^{3} q}{(2 \pi)^{3}} \mathcal{M}_{s}^{2}(q) P_{\phi}(q) q^{n_{f}} \\
& =\frac{1}{\sigma_{0 s}^{2}} \frac{f_{\mathrm{NL}}\left(k_{p}\right)}{k_{p}^{n_{f}}} \sigma_{\alpha s}^{2}
\end{aligned}
$$

where $\sigma_{\alpha s}$ is the spectral moment evaluated for $\alpha=n_{f} / 2$. Therefore, the non-Gaussian correction to the peak power spectrum becomes

$$
\Delta P_{>\nu}(k) \stackrel{k \rightarrow 0}{=} 4 c_{1} c_{2} f_{\mathrm{NL}}\left(k_{p}\right) k_{p}^{-n_{f}} \sigma_{\alpha s}^{2} \mathcal{M}_{s}^{-1}(k) P_{s}(k) .
$$

This result agrees with that of $[14,18]$ in the high-peak limit only, for which $c_{1} c_{2} \approx\left(\nu / \sigma_{0 s}\right)^{3}$.

Finally, for the folded, orthogonal, and equilateral bispectrum shapes, the power spectrum of thresholded regions is also given by Eq. (51), with $\mathcal{F}_{s}^{(3)}$ obtained from an integration over Eqs. (6) and (8), respectively [31]. In the limit $k \ll 1$, we can set $\mathcal{M}_{s}(q) \approx \mathcal{M}_{s}\left(k_{1}\right)$ and, on expanding $P_{\phi}(q)$ at second-order in $k / k_{1}$, we arrive at

$$
\Delta P_{>\nu}(k) \stackrel{k \rightarrow 0}{=} 6 A c_{1} c_{2} f_{\mathrm{NL}} \sigma_{\alpha s}^{2} k^{-2 \alpha} \mathcal{M}_{s}(k) P_{\phi}(k),
$$

with $A=1, \quad \alpha=\left(n_{s}-4\right) / 6 \approx-1 / 2$ (folded shape), $A=-2, \alpha=\left(n_{s}-4\right) / 6 \approx-1 / 2$ (orthogonal shape), and $A=2, \alpha=\left(n_{s}-4\right) / 3 \approx-1$ (equilateral shape). Again, we recover the high-peak expression [4] if we take the limit $\nu \rightarrow \infty$. 


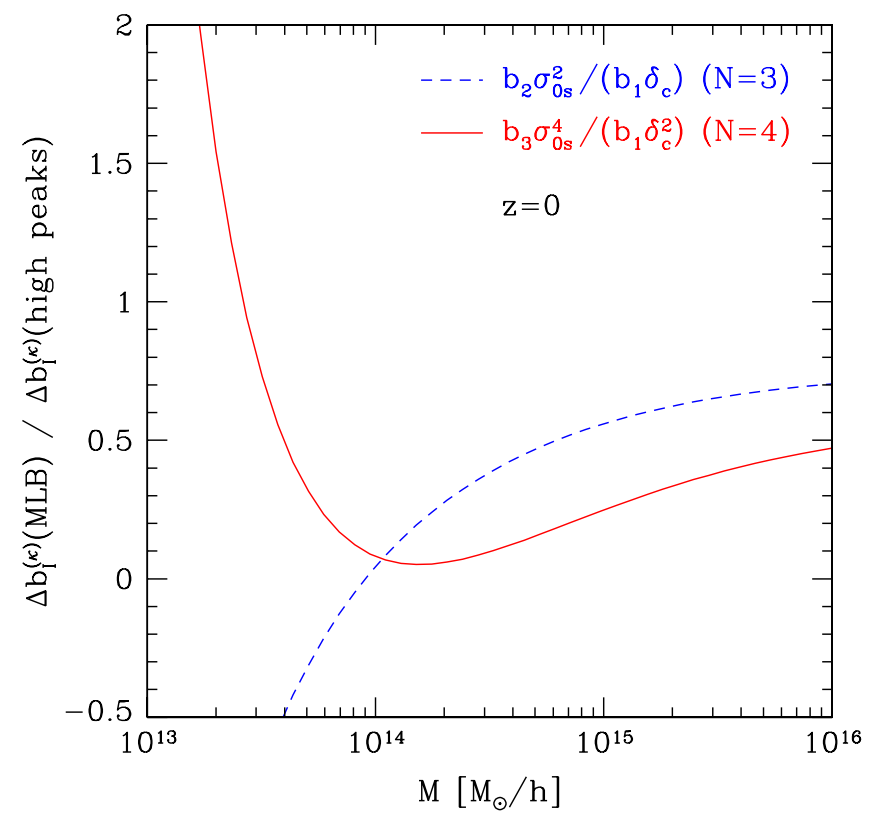

FIG. 1 (color online). Ratio of the non-Gaussian correction to the linear bias predicted by the statistics of thresholded regions to that obtained in the high-peak limit. For a nonzero primordial bispectrum $(N=3)$ and trispectrum $(N=4)$, this ratio is equal to $b_{2} \sigma_{0 s}^{2} /\left(b_{1} \delta_{c}\right)$ and $b_{3} \sigma_{0 s}^{4} /\left(b_{1} \delta_{c}^{2}\right)$, respectively. Note that it depends on the order $N$ but not on the specific shape of the primordial correlation function. Results are shown at $z=0$ as a function of halo mass $M$. The Gaussian bias parameters $b_{N}$ are computed from a Sheth-Tormen mass function.

\section{Beyond the high-peak limit}

Figure 1 shows the ratio of the non-Gaussian linear bias correction arising from the statistics of thresholded regions to that obtained in the high-peak approximation. In the literature, $c_{N}$ is conventionally replaced with $b_{N}$, so that this ratio becomes $b_{2} \sigma_{0 s}^{2} /\left(b_{1} \delta_{c}\right)$ for a primordial bispectrum, and $b_{3} \sigma_{0 s}^{4} /\left(b_{1} \delta_{c}^{2}\right)$ for a primordial trispectrum. Note that these quantities do not depend on the shape of the polyspectrum considered. The results are shown at $z=0$ using the Gaussian bias factors $b_{N}$ derived from the ShethTormen mass function $[48,49]$ with $p=0.3$ and $q=0.75$, via the PBS approach. These predictions are clearly at odds with the simulation results: first, for the local bispectrum shape with constant $f_{\mathrm{NL}}$, there is no evidence of a large suppression relative to the high-peak expression [8-10,50] (the correction factor of $\sim 0.8$ advocated by $[9,10]$ likely applies for friends-of-friends halo finders solely; see [4] for a discussion). Second, the simulation studies of $[14,15]$ unambiguously show that the correction to the high-peak expression depends on the shape of the bispectrum. Third, while the suppression seen in Fig. 1 for $M \gtrsim 10^{14} \mathrm{M}_{\odot} / \mathrm{h}$ is qualitatively consistent with that measured for the $g_{\mathrm{NL}} \phi^{3}$ model for highly biased halos [13], the sharp upturn below $\sim 10^{13} \mathrm{M}_{\odot} / \mathrm{h}$ is inconsistent with the findings of [13] at high significance. This appears to exclude the statistics of thresholded regions and, more generally, local bias expansions of the form Eq. (31) as a viable framework to calculate non-Gaussian bias corrections, at least for realistic halo masses $\left(\lesssim 10^{15} M_{\odot}\right)$. We return to these issues in Sec. VI.

\section{PEAK-BACKGROUND SPLIT: SEPARATION OF SCALES}

In this section, we present our second derivation of the non-Gaussian, scale-dependent halo bias, based on the peak-background split argument. We show that the fact that the cumulants of the density field depend on the smoothing scale $R_{s}$ induces an important and previously overlooked contribution to the non-Gaussian bias correction.

In this approach, we make a separation of scales and split all perturbations $\delta, \phi$, etc., into their long-wavelength (subscript " $L$ ") and short-wavelength (subscript " $S$ ") pieces, e.g.,

$$
\delta=\delta_{L}+\delta_{S}, \quad \phi=\phi_{L}+\phi_{S}, \quad \ldots
$$

Here, short wavelengths signify the scales which impact halo formation $(\leq 10-100 \mathrm{Mpc} / h)$, while long wavelengths correspond to the scales on which we would like to measure the clustering of halos $(\gtrsim 100 \mathrm{Mpc} / h)$. For a Gaussian density field with independent Fourier modes, the $L$ and $S$ pieces are statistically independent. In the presence of NG, this is no longer the case. As we will see shortly, it will be convenient to apply the peakbackground split to the Gaussian primordial curvature perturbation $\phi$. This approach isolates the effect of mode-coupling introduced by primordial NG, allowing for direct physical insights. To avoid confusion, we shall denote the physical, non-Gaussian density field by $\hat{\delta}$, to distinguish it from the Gaussian density field $\delta$ related to the Gaussian potential $\phi$.

\section{A. General cubic NG}

Consider the case of weakly non-Gaussian potential perturbations described via nonzero three- and four-point functions. We can capture the non-Gaussian corrections by generalizing the cubic local ansatz Eq. (2) in Fourier space:

$$
\begin{aligned}
\Phi(\mathbf{k})= & \phi(\mathbf{k})+f_{\mathrm{NL}} \int \frac{d^{3} k_{1}}{(2 \pi)^{3}} \int \frac{d^{3} k_{2}}{(2 \pi)^{3}} \omega^{(2)}\left(\mathbf{k}_{1}, \mathbf{k}_{2}\right) \phi\left(\mathbf{k}_{1}\right) \phi\left(\mathbf{k}_{2}\right) \delta_{D}\left(\mathbf{k}-\mathbf{k}_{12}\right) \\
& +g_{\mathrm{NL}} \int \frac{d^{3} k_{1}}{(2 \pi)^{3}} \int \frac{d^{3} k_{2}}{(2 \pi)^{3}} \int \frac{d^{3} k_{3}}{(2 \pi)^{3}} \omega^{(3)}\left(\mathbf{k}_{1}, \mathbf{k}_{2}, \mathbf{k}_{3}\right) \phi\left(\mathbf{k}_{1}\right) \phi\left(\mathbf{k}_{2}\right) \phi\left(\mathbf{k}_{3}\right) \delta_{D}\left(\mathbf{k}-\mathbf{k}_{123}\right),
\end{aligned}
$$


where $\mathbf{k}_{12 \ldots}=\mathbf{k}_{1}+\mathbf{k}_{2}+\ldots$, and the two kernels $\omega^{(2)}$, $\omega^{(3)}$, are related to the three- and four-point function, respectively [18]. The relation is in general ambiguous, i.e., different kernels can yield the same three- and fourpoint functions. However, the large-scale limit of the nonGaussian bias depends on the squeezed limit of the $N$-point functions, as we have seen in Sec. III. In this limit, the kernels $\omega^{(2)}, \omega^{(3)}$ are unique [51].

One possible choice of kernels, which has the nice property (for analytical calculations) of being fully symmetric, is

$$
\begin{array}{r}
\omega^{(2)}\left(\mathbf{k}_{1}, \mathbf{k}_{2}\right)=\frac{1}{2 f_{\mathrm{NL}}} \frac{\xi_{\Phi}^{(3)}\left(\mathbf{k}_{1}, \mathbf{k}_{2}, \mathbf{k}_{3}\right)}{P_{1} P_{2}+2 \text { perm. }} \\
\omega^{(3)}\left(\mathbf{k}_{1}, \mathbf{k}_{2}, \mathbf{k}_{3}\right)=\frac{1}{6 g_{\mathrm{NL}}} \frac{\xi_{\Phi}^{(4)}\left(\mathbf{k}_{1}, \mathbf{k}_{2}, \mathbf{k}_{3}, \mathbf{k}_{4}\right)}{P_{1} P_{2} P_{3}+3 \text { perm. }},
\end{array}
$$

where in the first line, $k_{3}=\left|\mathbf{k}_{12}\right|$, while in the second line, $k_{4}=\left|\mathbf{k}_{123}\right|$, and $P_{i} \equiv P_{\phi}\left(k_{i}\right)$. We have left out the coefficients $f_{\mathrm{NL}}$ and $g_{\mathrm{NL}}$ for convenience. Note that, in general, the four-point function also contains terms of order $f_{\mathrm{NL}}^{2}$, which we assume to be included in $\xi_{\Phi}^{(4)}$ even though we parameterize the amplitude by a single coefficient $g_{\mathrm{NL}}$.

Equation (58) is analogous to the kernel $\tilde{W}\left(\mathbf{k}_{1}, \mathbf{k}_{2}\right)$ defined in [18], and Eq. (59) is the straightforward generalization to the cubic case. Note that we define the kernels in terms of $\phi$ here, while they are defined in terms of $\phi_{0}(k) \equiv T(k) \phi(k)$ in [18]. The final result (in the largescale limit) is independent of this choice of kernel, which yields $\omega^{(2)}=\omega^{(3)}=1$ for the local model.

In the next subsection, we first calculate the effect of long-wavelength perturbations $\phi_{L}, \delta_{L}$ on the statistics of the small-scale density field $\delta_{S}$. We then derive expressions for the non-Gaussian halo bias for general cubic NG.

\section{Effect of long-wavelength perturbations on the density field}

We begin by applying the separation of scales, Eq. (56), to Eq. (57). Clearly, for the quadratic part we will obtain the combinations $(S S),(S L)$, and $(L L)$, while the cubic part yields $(S S S),(S S L),(S L L)$, and $(L L L)$. The terms involving $L$ solely do not influence halo abundance (since they do not contribute significantly to the moments of the smallscale density field). The terms involving $S$-perturbations only increase the variance, skewness, and kurtosis of the small-scale density field. They may thus affect the abundance of halos. However, they do so in a scale-independent way and, thereby, induce at most a scale-independent bias correction. Hence, in order to derive the (scale-dependent) effect of NG on halo clustering, we only need to retain the mixed terms

We now want to derive an expression for the nonGaussian small-scale density field $\hat{\delta}_{S}(k)=\mathcal{M}(k) \Phi_{S}$. We obtain it by multiplying the short-wavelength part of Eq. (57) by $\mathcal{M}(k)$. Next, we apply a trick, noting that $\mathcal{M}(k) \propto k^{2}$, and $k^{2}=\left[\mathbf{k}_{1}+\left(\mathbf{k}-\mathbf{k}_{1}\right)\right]^{2}$. Thus,

$$
\mathcal{M}(k)=\mathcal{M}\left(k_{1}\right)+\mathcal{M}\left(\left|\mathbf{k}-\mathbf{k}_{1}\right|\right)+\mathcal{O}\left(\mathbf{k}_{1} \cdot\left[\mathbf{k}-\mathbf{k}_{1}\right]\right) .
$$

When inserting Eq. (60) into the first line of Eq. (57), we see that for the local model, where $\omega^{(2)}=1$, the last term in Eq. (60) corresponds to the Fourier transform of $\nabla \phi_{L}$. $\nabla \phi_{S}$ (recall that we are only dealing with mixed terms). When averaging over a region where $\nabla \phi_{L}$ is approximately a constant gradient, this term vanishes since $\phi_{S}$ is uncorrelated with $\phi_{L}$ (see also [7] for a different procedure in the local case). Below we will perform precisely such an averaging procedure. A similar reasoning can be applied to the nonlocal case. Hence, we will drop this term and its analogs in the cubic part of Eq. (57). Note that we have neglected the $k$-dependence of the transfer function here. One can circumvent this by defining the kernel in terms of $\phi_{0}$, as done in [18]. Equation (60) and its generalization to several $k_{i}$ s then lead to

$$
\begin{aligned}
\hat{\delta}_{S}(\mathbf{k})= & \delta_{S}(\mathbf{k})+2 f_{\mathrm{NL}} \int \frac{d^{3} k_{1}}{(2 \pi)^{3}} \omega^{(2)}\left(\mathbf{k}_{1}, \mathbf{k}-\mathbf{k}_{1}\right)\left[\delta_{L}\left(\mathbf{k}_{1}\right) \phi_{S}\left(\mathbf{k}-\mathbf{k}_{1}\right)+\delta_{S}\left(\mathbf{k}_{1}\right) \phi_{L}\left(\mathbf{k}-\mathbf{k}_{1}\right)\right] \\
& +3 g_{\mathrm{NL}} \int \frac{d^{3} k_{1}}{(2 \pi)^{3}} \int \frac{d^{3} k_{2}}{\left(2 \pi^{3}\right)} \omega^{(3)}\left(\mathbf{k}_{1}, \mathbf{k}_{2}, \mathbf{k}-\mathbf{k}_{12}\right)\left[2 \phi_{L} \delta_{L} \phi_{S}+\phi_{L} \phi_{L} \delta_{S}+2 \phi_{L} \phi_{S} \delta_{S}+\delta_{L} \phi_{S} \phi_{S}\right] .
\end{aligned}
$$

In the second line, we have omitted the arguments of $\phi, \delta$ for brevity (the factors in each product are evaluated at $\mathbf{k}_{1}$, $\mathbf{k}_{2}$, and $\mathbf{k}-\mathbf{k}_{12}$, respectively).

In the presence of NG, the statistical properties of $\hat{\delta}_{S}$ can be derived straightforwardly from Eq. (61) by taking advantage of the fact that $\phi_{S}, \delta_{S}$ are Gaussian fields. We will consider a region of "intermediate" size $R \gg R_{S}$ over which the long-wavelength perturbations can be considered constant. This approximation will break down when predicting the clustering on scales which contribute significantly to $\sigma_{0 s}$ (see the discussion below and in [18]). We then calculate the variance and skewness of $\hat{\delta}_{S}$ in the presence of "external" perturbations $\phi_{L}, \delta_{L}$. To compute the variance, for instance, we calculate $\left\langle\hat{\delta}_{S}(\mathbf{k}) \hat{\delta}_{S}\left(\mathbf{k}^{\prime}\right)\right\rangle$ and integrate over $\mathbf{k}$. It is sufficient to consider a single (for the quadratic terms) or two independent (for the cubic terms) 
long-wavelength Fourier modes and, hence, omit the integrals over $k_{1}, k_{2}$. This is because we will eventually take derivatives with respect to single long-wavelength Fourier modes in order to derive the non-Gaussian scale-dependent bias. The variance on scale $R_{s}$ reads

$$
\begin{aligned}
\hat{\sigma}_{0 s}^{2} \equiv & \left\langle\hat{\delta}_{S, R_{s}} \hat{\delta}_{S, R_{s}}\right\rangle_{R} \\
= & \sigma_{0 s}^{2}+4 f_{\mathrm{NL}}\left[\phi_{L}(\mathbf{k}) \sigma_{\omega s}^{2}(k)+\delta_{L}(\mathbf{k}) \sigma_{\omega \phi s}^{2}(k)\right] \\
& +6 g_{\mathrm{NL}} \phi_{L}\left(\mathbf{k}_{1}\right) \phi_{L}\left(\mathbf{k}_{2}\right) \sigma_{\omega s}^{2}\left(\mathbf{k}_{1}, \mathbf{k}_{2}\right) \\
& +6 g_{\mathrm{NL}}\left[\phi_{L}\left(\mathbf{k}_{1}\right) \delta_{L}\left(\mathbf{k}_{2}\right)+\phi_{L}\left(\mathbf{k}_{2}\right) \delta_{L}\left(\mathbf{k}_{1}\right)\right] \sigma_{\omega \phi s}^{2}\left(\mathbf{k}_{1}, \mathbf{k}_{2}\right),
\end{aligned}
$$

where $\langle\cdot\rangle_{R}$ indicates an average over a given intermediate region of size $R$. Note that the terms from quadratic NG are linear in $\phi_{L}, \delta_{L}$, while those from cubic NG are quadratic in $\phi_{L}, \delta_{L}$. For Eq. (62), we have defined the following $k$-dependent spectral moments (not to be confounded with Eq. (44), which does not depend on $k$ ):

$$
\begin{gathered}
\sigma_{\omega s}^{2}(k) \equiv \int \frac{d^{3} k_{s}}{(2 \pi)^{3}} \omega^{(2)}\left(\mathbf{k}, \mathbf{k}_{s}\right) \mathcal{M}_{s}^{2}\left(k_{s}\right) P_{\phi}\left(k_{s}\right) \\
\sigma_{\omega \phi s}^{2}(k) \equiv \int \frac{d^{3} k_{s}}{(2 \pi)^{3}} \omega^{(2)}\left(\mathbf{k}, \mathbf{k}_{s}\right) \mathcal{M}_{s}\left(k_{s}\right) P_{\phi}\left(k_{s}\right) \\
\sigma_{\omega s}^{2}\left(\mathbf{k}_{1}, \mathbf{k}_{2}\right) \equiv \int \frac{d^{3} k_{s}}{(2 \pi)^{3}} \omega^{(3)}\left(\mathbf{k}_{1}, \mathbf{k}_{2}, \mathbf{k}_{s}\right) \mathcal{M}_{s}^{2}\left(k_{s}\right) P_{\phi}\left(k_{s}\right) \\
\sigma_{\omega \phi s}^{2}\left(\mathbf{k}_{1}, \mathbf{k}_{2}\right) \equiv \int \frac{d^{3} k_{s}}{(2 \pi)^{3}} \omega^{(3)}\left(\mathbf{k}_{1}, \mathbf{k}_{2}, \mathbf{k}_{s}\right) \mathcal{M}_{s}\left(k_{s}\right) P_{\phi}\left(k_{s}\right) .
\end{gathered}
$$

In the following, we will ignore the term $4 f_{\mathrm{NL}} \delta_{L} \sigma_{\omega \phi s}^{2}$ since it only generates a very small $\left(\lesssim 10^{-4} f_{\mathrm{NL}}\right)$ scaleindependent correction to the halo bias.

At cubic-order in Eq. (61), there are two terms of the type $(L S S)$. These terms indicate that the small-scale density acquires a skewness (third moment) which is modulated by long-wavelength perturbations. We will only include the effect of the first term, $6 g_{\mathrm{NL}} \phi_{L} \phi_{S} \delta_{S}$, as the second term proportional to $\delta_{L} \phi_{S}^{2}$ only produces a scaleindependent correction to the halo bias. The three-point function of the small-scale density field induced by a single long-wavelength perturbation $\phi_{L}\left(\mathbf{k}_{l}\right)$ is given by

$$
\begin{aligned}
\langle\delta(\mathbf{k}) & \left.\delta\left(\mathbf{k}^{\prime}\right) \delta\left(\mathbf{k}^{\prime \prime}\right)\right\rangle_{R} \\
= & 3 g_{\mathrm{NL}} \phi_{L}\left(\mathbf{k}_{l}\right)(2 \pi)^{3} \delta_{D}\left(\mathbf{k}+\mathbf{k}^{\prime}+\mathbf{k}^{\prime \prime}\right) \mathcal{M}(k) \mathcal{M}\left(k^{\prime}\right) \mathcal{M}\left(k^{\prime \prime}\right) \\
& \times\left\{\left[\omega^{(3)}\left(\mathbf{k}_{l}, \mathbf{k}^{\prime}, \mathbf{k}^{\prime \prime}\right)+\omega^{(3)}\left(\mathbf{k}_{l}, \mathbf{k}^{\prime \prime}, \mathbf{k}^{\prime}\right)\right]\right. \\
& \left.\times P_{\phi}\left(k^{\prime}\right) P_{\phi}\left(k^{\prime \prime}\right)+2 \text { perm }\right\},
\end{aligned}
$$

where " 2 perm" indicates the two cyclic permutations of $\left(k, k,^{\prime} k^{\prime \prime}\right)$. Recall that the subscript $R$ on the expectation value indicates averaging over a region where $\phi_{L}$ is approximately constant. In deriving Eq. (67), we have used that $k=\left|\mathbf{k}^{\prime}+\mathbf{k}^{\prime \prime}\right|$, so that $\mathcal{M}\left(k^{\prime}\right)+\mathcal{M}\left(k^{\prime \prime}\right) \approx \mathcal{M}(k)$ on large scales. Thus, the three-point function of the smallscale density field induced by a long-wavelength perturbation in cubic NG is equivalent to that arising in a quadratic model of NG described by the effective three-point function

$$
\begin{aligned}
\xi_{\Phi, \mathrm{eff}}^{(3)} & \left(\mathbf{k}, \mathbf{k}^{\prime}, \mathbf{k}^{\prime \prime}\right) \\
= & f_{\mathrm{NL}, \mathrm{eff}}\left(\mathbf{k}_{l}\right)\left\{\left[\omega^{(3)}\left(\mathbf{k}_{l}, \mathbf{k}^{\prime}, \mathbf{k}^{\prime \prime}\right)+\omega^{(3)}\left(\mathbf{k}_{l}, \mathbf{k}^{\prime \prime}, \mathbf{k}^{\prime}\right)\right]\right. \\
& \left.\times P_{\phi}\left(k^{\prime}\right) P_{\phi}\left(k^{\prime \prime}\right)+2 \mathrm{perm}\right\},
\end{aligned}
$$

where $f_{\mathrm{NL}, \text { eff }}=3 g_{\mathrm{NL}} \phi_{L}\left(\mathbf{k}_{l}\right)$. Note that $\xi_{\Phi, \text { eff }}^{(3)}$ generally depends on the scale $k_{l}$ of the long-wavelength perturbation. We can now calculate the skewness parameter of the small-scale non-Gaussian density field, taking out the scaling with the long-wavelength mode $\phi_{L}$ :

$$
\begin{aligned}
\hat{S}_{s}^{(3)} \equiv & \frac{\left\langle\hat{\delta}_{s, R_{s}}^{3}\right\rangle_{R}}{\left\langle\hat{\delta}_{s, R_{s}}^{2}\right\rangle_{R}^{2}}=3 g_{\mathrm{NL}} \phi_{L}\left(\mathbf{k}_{l}\right) S_{\omega s}^{(3)}\left(k_{l}\right), \\
S_{\omega s}^{(3)}\left(k_{l}\right) \equiv & \frac{6}{\sigma_{0 s}^{4}} \int \frac{d^{3} k_{1}}{\left(2 \pi^{3}\right)} \mathcal{M}_{s}\left(k_{1}\right) P_{\phi}\left(k_{1}\right) \\
& \times \int \frac{d^{3} k_{2}}{\left(2 \pi^{3}\right.} \mathcal{M}_{s}\left(k_{2}\right) P_{\phi}\left(k_{2}\right) \\
& \times \omega^{(3)}\left(\mathbf{k}_{l}, \mathbf{k}_{1}, \mathbf{k}_{2}\right) \mathcal{M}_{s}\left(\left|\mathbf{k}_{1}+\mathbf{k}_{2}\right|\right) .
\end{aligned}
$$

Here, we have noted that $\left\langle\hat{\delta}_{s}^{3}\right\rangle$ is already linear in $g_{\mathrm{NL}}$, so that we can set $\left\langle\hat{\delta}_{s}^{2}\right\rangle=\sigma_{0 s}^{2}$.

Summarizing, the effect of long-wavelength modes in general cubic NG is to rescale the local small-scale variance of the density field [Eq. (61)], as was discussed for the quadratic case in $[3,6,18]$. This rescaling is linear in the long-wavelength modes for the quadratic $\left(f_{\mathrm{NL}}\right)$ term, and quadratic in $\delta_{L}, \phi_{L}$ for the cubic $\left(g_{\mathrm{NL}}\right)$ term. The terms quadratic in the $L$-modes induce a non-Gaussian correction to the second-order bias $b_{\text {II }}$. We will not consider this correction here as it does not significantly impact the halo power spectrum. Furthermore, a long-wavelength mode in a cubic model also induces a local three-point function (skewness) in the density field [Eq. (67)]: observers in a region with $\phi_{L} \neq 0$ see a local Universe with an effective quadratic NG described by the "primordial" three-point function $\xi_{\Phi, \text { eff }}^{(3)}$ [Eq. (68)].

\section{Non-Gaussian corrections to the linear bias}

Let us now consider the halo abundance $\hat{n}_{h}(\mathbf{x})$ in some region of size $R$, with $R_{s} \ll R \ll R_{l}$, and $R_{s}$ being the Lagrangian scale associated with a halo mass $M$. Throughout, we will assume that $\hat{n}_{h}$ depends only on the matter density $\rho_{R}$ averaged over $R$, and the moments of the small-scale fluctuations: $\hat{\sigma}_{0 s}^{2}, \hat{S}_{s}^{(3)}$, and $\cdots$. While, in 
the Gaussian case, a perturbation $\delta_{L}$ only changes the average density $\left[\rho_{R} \rightarrow \rho_{R}\left(1+\delta_{L}\right)\right]$, it also affects all the cumulants of the density field when the initial conditions are non-Gaussian. Applying the chain rule, we find

$$
\begin{aligned}
\mathrm{b}_{\mathrm{I}}(k) & \left.\equiv \frac{1}{\overline{\bar{n}}_{h}} \frac{d \hat{\bar{n}}_{h}}{d \delta_{L}(k)}\right|_{\delta_{L}=0} \\
& =\frac{\partial \ln \hat{\bar{n}}_{h}}{\partial \ln \rho_{R}}+\frac{\partial \ln \hat{\bar{n}}_{h}}{\partial \ln \hat{\sigma}_{0 s}} \frac{\partial \ln \hat{\sigma}_{0 s}}{\partial \delta_{L}(k)}+\frac{\partial \ln \hat{\bar{n}}_{h}}{\partial \hat{S}_{s}^{(3)}} \frac{\partial \hat{S}_{s}^{(3)}}{\partial \delta_{L}(k)}+\cdots
\end{aligned}
$$

Here, $\hat{\bar{n}}_{h}$ is the average number density of halos of mass $M$ with non-Gaussian initial conditions, and all derivatives are evaluated at $\delta_{L}=0$. Owing to isotropy, $\mathrm{b}_{\mathrm{I}}(k)$ only depends on the magnitude of the $k$-vector. The first term in Eq. (71) is the usual Gaussian bias $b_{1}$, while the second and third terms yield the non-Gaussian corrections. Thus, the nonGaussian contribution $\Delta \mathrm{b}_{\mathrm{I}}(k)$ to the linear bias $\mathrm{b}_{\mathrm{I}}(k)$ arises from the dependence of the halo abundance on the variance and skewness of the density field.

Let us deal with the variance first. As we have seen in the last section, the change in the variance from cubic NG is $\mathcal{O}\left(\delta_{L}^{2}\right)$. Hence, these terms do not contribute to the linear bias and Eq. (61) gives

$$
\left.\frac{\partial \ln \hat{\sigma}_{0 s}}{\partial \delta_{L}(k)}\right|_{\delta_{L}=0}=2 f_{\mathrm{NL}} \frac{\sigma_{\omega s}^{2}(k)}{\sigma_{0 s}^{2}} \mathcal{M}^{-1}(k) .
$$

Note that this expression in general depends on the smoothing scale $R_{S}$ or, equivalently, the halo mass $M$.

To proceed further, we will restrict ourselves to the case of a universal mass function for Gaussian initial conditions. Therefore, the Gaussian halo number density is given by Eq. (11). Throughout this section, we will not need to specify $f(\nu)$ explicitly. The non-Gaussian halo abundance $\hat{\bar{n}}_{h}$ will thus depend on $\hat{\sigma}_{0 s}$ through the significance $\nu=$ $\delta_{c} / \hat{\sigma}_{0 s}$ and the Jacobian $\partial \hat{\sigma}_{0 s} / \partial \ln M$. Noting that the Gaussian bias is $b_{1}=-\delta_{c}^{-1} d \ln f(\nu) / d \ln \nu$, and taking the derivative of Eq. (72) with respect to $\ln M$, we obtain

$$
\begin{aligned}
\left.\frac{\partial \ln \hat{\bar{n}}_{h}}{\partial \ln \sigma_{0 s}} \frac{\partial \ln \hat{\sigma}_{0 s}}{\partial \delta_{L}(k)}\right|_{\delta_{L}=0}= & 2 f_{\mathrm{NL}} \mathcal{M}^{-1}(k) \frac{\sigma_{\omega s}^{2}(k)}{\sigma_{0 s}^{2}} \\
& \times\left[b_{1} \delta_{c}+2 \varepsilon_{\omega s}(k)\right], \\
\varepsilon_{\omega s}(k) \equiv & \frac{\partial \ln \sigma_{\omega s}^{2}(k)}{\partial \ln \sigma_{0 s}^{2}}-1 .
\end{aligned}
$$

The second term in the square brackets, $2 \varepsilon_{\omega s}(k)$, has previously been neglected [14,18]. It vanishes in the scale-independent local model, for which $\omega^{(2)}=1$ and $\sigma_{\omega s}=\sigma_{0 s}$, but is nonzero and generally significant for other bispectrum shapes. Physically, this term comes about because a scale-dependent rescaling of the variance [Eq. (72)] also changes the significance interval $d \nu$ that corresponds to a fixed mass interval $d M$. This in turn affects the abundance of halos at a fixed mass and thus contributes to the non-Gaussian bias. The term is absent in the results of the thresholding approach (Sec. III), since the cumulative two-point correlation $\xi_{>\nu}(r)$ is computed at a fixed smoothing scale $R_{s}$. We return to this point in Sec. VI.

In order to derive the effect of cubic NG, we need to determine the dependence of $\hat{\bar{n}}_{h}$ on $\hat{S}_{s}^{(3)}$, i.e., the effect of a primordial three-point function on the average abundance of halos. Different (albeit related) expressions have been proposed for the change in the halo abundance induced by primordial NG [52-59]. For definiteness, we will adopt the prescription of [53] derived from an Edgeworth expansion of $P_{1}(>\nu)$ (see also Sec. V),

$$
\begin{aligned}
\hat{\bar{n}}_{h}\left(\rho, \hat{\sigma}_{0 s}, \hat{S}_{s}^{(3)}\right) & =\bar{n}_{h}\left(\rho, \hat{\sigma}_{0 s}, 0\right)\left[1+\frac{1}{6} \hat{\sigma}_{0 s} \hat{S}_{s}^{(3)}\left(\nu^{3}-3 \nu\right)+\frac{1}{6} \frac{\partial\left(\hat{\sigma}_{0 s} \hat{S}_{s}^{(3)}\right)}{\partial \ln \hat{\sigma}_{0 s}}\left(\nu-\frac{1}{\nu}\right)\right] \\
& =\bar{n}_{h}\left(\rho, \hat{\sigma}_{0 s}, 0\right)\left\{1+\frac{1}{6} \hat{\sigma}_{0 s} \hat{S}_{s}^{(3)}\left[\left(\nu^{3}-3 \nu\right)+\left(1+\frac{\partial \ln \hat{S}_{s}^{(3)}}{\partial \ln \hat{\sigma}_{0 s}}\right)\left(\nu-\frac{1}{\nu}\right)\right]\right\} .
\end{aligned}
$$

In principle however, any other prescription for the response of halo number counts to a small-scale skewness of the density field could be employed here. From Eq. (75), we derive

$$
\left.\frac{6}{\sigma_{0 s}^{2}} \frac{\partial \ln \hat{\bar{n}}_{h}}{\partial \hat{S}_{s}^{(3)}}\right|_{\delta_{L}=0}=\frac{1}{\sigma_{0 s}}\left[\left(\nu^{3}-3 \nu\right)+\left.\left(1+\frac{\partial \ln \hat{S}_{s}^{(3)}}{\partial \ln \hat{\sigma}_{0 s}}\right)\right|_{\delta_{L}=0}\left(\nu-\frac{1}{\nu}\right)\right]=b_{2} \delta_{c}+\left(1+\frac{\partial \ln \hat{S}_{s}^{(3)}}{\partial \ln \sigma_{0 s}}\right) b_{1} .
$$

In the last equality, we have identified the $\nu$-polynomials with the Gaussian peak-background split biases derived from the multiplicity function $f(\nu)=$ $\sqrt{2 / \pi} \nu \exp \left(-\nu^{2} / 2\right)$, since our parameterization of $\hat{\bar{n}}_{h}$ in terms of $\hat{S}^{(3)}$ was derived within the Press-Schechter formalism [53]. While for high peaks $\nu \gg 1$, the first term in the last equality will dominate, for more abundant halos the second term can contribute significantly. The latter again arises because of the dependence of $S_{\omega s}^{(3)}$ on the smoothing scale $R_{s}$.

Finally, using Eq. (69) we find

$$
\left.\frac{\partial \hat{S}_{s}^{(3)}}{\partial \delta_{L}(k)}\right|_{\delta_{L}=0}=3 g_{\mathrm{NL}} \mathcal{M}^{-1}(k) S_{\omega s}^{(3)}(k)
$$


Then, using Eq. (71) together with Eqs. (73) and (76), we can assemble the expression for the scale-dependent halo bias in a general, cubic-order model of NG:

$$
\begin{aligned}
\Delta \mathrm{b}_{\mathrm{I}}^{(\kappa)}(k)= & 2 f_{\mathrm{NL}} \mathcal{M}^{-1}(k) \frac{\sigma_{\omega s}^{2}(k)}{\sigma_{0 s}^{2}}\left[b_{1} \delta_{c}+2 \varepsilon_{\omega s}(k)\right] \\
& +\frac{1}{2} g_{\mathrm{NL}} \mathcal{M}^{-1}(k) \sigma_{0 s}^{2} S_{\omega s}^{(3)}(k) \\
& \times\left[b_{2} \delta_{c}+\left(1+\frac{\partial \ln S_{\omega s}^{(3)}(k)}{\partial \ln \sigma_{0 s}}\right) b_{1}\right] .
\end{aligned}
$$

The superscript $(\kappa)$ emphasizes that this correction is $k$-dependent, and distinguishes it from a $k$-independent non-Gaussian bias which we shall denote with a superscript $(\iota)$. Note that the terms in the first line of Eq. (78) apply for any universal mass function prescription. On the other hand, the coefficients in the square brackets of the second line will change if a different prescription for $\partial \ln \hat{\bar{n}}_{h} / \partial \hat{S}_{s}^{(3)}$ is adopted.

\section{B. Application to models of NG}

\section{Local NG}

In the local model [Eq. (2)], the kernels Eqs. (58) and (59) are simply $\omega^{(2)}=\omega^{(3)}=1$. Thus, $\sigma_{\omega s} \rightarrow \sigma_{0 s}$, and the skewness $S_{\omega s}^{(3)}$ induced by a long-wavelength perturbation becomes $S_{s, \text { loc }}^{(3)}$, i.e., the skewness in a local quadratic model with $f_{\mathrm{NL}}=1$ [Eq. (49)]. A more direct way to derive this result is to note that the $(L S S)$ terms in the second line of Eq. (61) are obtained by applying the Poisson equation to an effective non-Gaussian potential

$$
\hat{\phi}_{S}=\phi_{S}+\left(3 g_{\mathrm{NL}} \phi_{L}\right) \phi_{S}^{2} \text {. }
$$

This relation tells us that, in the presence of cubic local NG, a region with a long-wavelength perturbation $\phi_{L}$ looks like a Universe with a local quadratic $f_{\mathrm{NL}}=3 g_{\mathrm{NL}} \phi_{L}$.

Since $\varepsilon_{\omega s}=0$, the correction to the first-order bias Eq. (78) then simplifies to

$$
\Delta \mathrm{b}_{\mathrm{I}}^{(\kappa)}(k)=\left[2 f_{\mathrm{NL}} b_{1} \delta_{c}+\frac{1}{2} g_{\mathrm{NL}} \sigma_{0 s}^{2} S_{s, \mathrm{loc}}^{(3)} \epsilon_{S}\right] \mathcal{M}^{-1}(k),
$$

where we have defined

$$
\epsilon_{S} \equiv b_{2} \delta_{c}+\left(1+\frac{\partial \ln S_{s, \mathrm{loc}}^{(3)}}{\partial \ln \sigma_{0 s}}\right) b_{1} .
$$

The term linear in $f_{\mathrm{NL}}$ recovers the well-known result for the local quadratic model (this is due to the fact that $\hat{\sigma}_{0 s} / \sigma_{0 s}=1+2 f_{\mathrm{NL}} \phi_{L}$ is scale-independent). However, the term linear in $g_{\mathrm{NL}}$ departs from the high-peak expression derived in [13] as it includes a correction involving the logarithmic slope of $S_{s, \text { loc }}^{(3)}$ on $\sigma_{0 s}$. We will return to this point in Sec. VI. In the range $R_{s} \sim 1-10 \mathrm{~h}^{-1} \mathrm{Mpc}$, the scale-dependence of $S_{s, \text { loc }}^{(3)}$ is accurately reproduced by an

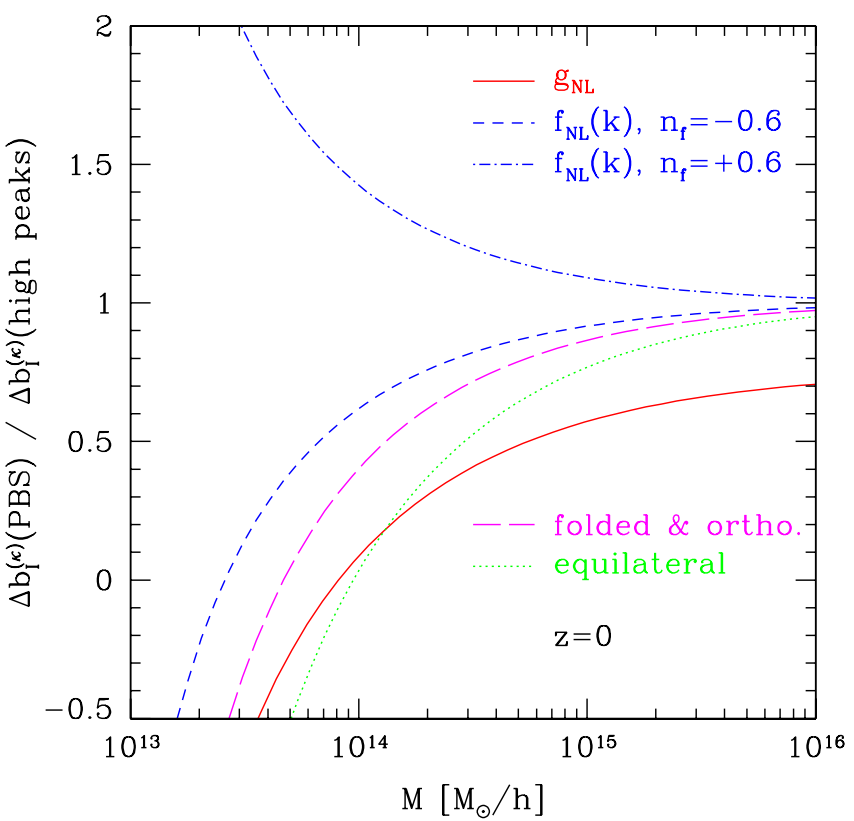

FIG. 2 (color online). Ratio of the non-Gaussian correction to the linear bias predicted by the peak-background split approach to that obtained in the high-peak limit. Results are shown at $z=0$ as a function of the halo mass $M$ for a local trispectrum with cubic parameter $g_{\mathrm{NL}}$ (solid curve), a local bispectrum with $k$-dependent quadratic parameter $f_{\mathrm{NL}}$ and index $n_{f}= \pm 0.6$ (dashed and dot-dashed curve), the folded and orthogonal template (long-dashed curve) and the equilateral bispectrum shape (dotted curve). In contrast to Fig. 1, the ratio sensitively depends on the shape of the primordial $N$-point function.

empirical power-law relation, $S_{s, \text { loc }}^{(3)} \approx 3.08 \times 10^{-4} \hat{\sigma}_{0 s}^{-0.855}$ for our fiducial cosmology (this agrees with the findings of $[60,61])$. Hence, the second term in Eq. (81) is approximately $0.145 b_{1}$ and, therefore, not negligible.

The ratio of the peak-background split prediction to the high-peak result is given by $\hat{\sigma}_{0 s}^{2} \epsilon_{S} /\left(b_{1} \delta_{c}^{2}\right)$. In Fig. 2, the value of this ratio in the limit $k \rightarrow 0$ is shown as the solid curve. We assume a critical collapse density $\delta_{c}=1.69$ and, in the calculation of the Gaussian biases $b_{N}$, we employ again a Sheth-Tormen multiplicity function with $p=0.3$ and $q=0.75$. As can be seen, the ratio depends strongly on the halo mass $M$. At the redshift assumed here $(z=0)$, it reverses sign around $M \simeq 7 \times 10^{13} \mathrm{M}_{\odot} / \mathrm{h}$.

\section{Scale-dependent and nonlocal NG}

We now turn to the other models of primordial NG introduced in Sec. II. Since these are all quadratic models, we can ignore the term linear in $g_{\mathrm{NL}}$ in Eq. (78). The dependence of $\Delta \mathrm{b}_{\mathrm{I}}(k)$ on the shape of NG enters through the moment $\sigma_{\omega s}(k)$ [Eq. (63)] and through the new correction proportional to $\partial \ln \sigma_{\omega s}(k) / \partial \ln \sigma_{0 s}$. Since we are interested in the large-scale limit, we can use the scaling of the kernel $\omega^{(2)}$ in the squeezed limit in order to simplify the analytical expressions. For the local quadratic model with 
$k$-dependent $f_{\mathrm{NL}}$ [see Eq. (5)], the kernel in this limit reduces to

$$
\omega\left(\mathbf{k}, \mathbf{k}_{s}-\mathbf{k}\right) \stackrel{k \rightarrow 0}{=}\left(\frac{k_{s}}{k_{p}}\right)^{n_{f}}
$$

As a result,

$$
\sigma_{\omega s}^{2}(k)=\frac{\sigma_{\alpha s}^{2}}{k_{p}^{n_{f}}},
$$

where $\alpha \equiv n_{f} / 2$ and the spectral moment $\sigma_{\alpha s}$ is defined in Eq. (44) with $R=R_{s}$. Using the first line of Eq. (78), the non-Gaussian, scale-dependent bias correction is then given by

$$
\begin{aligned}
& \Delta b_{\mathrm{I}, \text { sc.loc. }}^{(\kappa)}(k) \\
& =2 f_{\mathrm{NL}}\left(k_{p}\right) k_{p}^{-n_{f}}\left(\frac{\sigma_{\alpha s}}{\sigma_{0 s}}\right)^{2}\left[b_{1} \delta_{c}+2\left(\frac{\partial \ln \sigma_{\alpha s}}{\partial \ln \sigma_{0 s}}-1\right)\right] \mathcal{M}^{-1}(k), \\
& \alpha=n_{f} / 2 .
\end{aligned}
$$

The logarithmic derivative of $\sigma_{\alpha s}$ w.r.t. $\sigma_{0 s}$ is always larger (smaller) than unity for $\alpha>0(\alpha<0)$, and reaches unity for $\alpha \neq 0$ only in the limit $\sigma_{0 s} \rightarrow 0$. For the folded and orthogonal bispectrum shapes [Eq. (6) and (7)], the kernel asymptotes to [18]

$$
\omega\left(\mathbf{k}, \mathbf{k}_{s}-\mathbf{k}\right) \stackrel{k \rightarrow 0}{=} \frac{3}{2} A\left(\frac{k_{s}}{k}\right)^{2 \alpha},
$$

with $2 \alpha=\left(n_{s}-1\right) / 3-1=\left(n_{s}-4\right) / 3 \approx-1$, and $A=1$ (folded) or $A=-2$ (orthogonal). Note that we have neglected corrections of order $\left(k / k_{s}\right)^{2}$ and higher here (although they are easy to include in a numerical calculation). Inserting this result into Eq. (78), we arrive at

$$
\begin{aligned}
& \Delta b_{\mathrm{I}, \mathrm{fol} / \mathrm{ort}}^{(\kappa)}(k) \\
& \quad=3 A f_{\mathrm{NL}}\left(\frac{\sigma_{\alpha s}}{\sigma_{0 s}}\right)^{2}\left[b_{1} \delta_{c}+2\left(\frac{\partial \ln \sigma_{\alpha s}}{\partial \ln \sigma_{0 s}}-1\right)\right] k^{-2 \alpha} \mathcal{M}_{s}^{-1}(k), \\
& \alpha=\left(n_{s}-4\right) / 6 .
\end{aligned}
$$

Finally, for the equilateral bispectrum, we have

$$
\omega\left(\mathbf{k}, \mathbf{k}_{s}-\mathbf{k}\right) \stackrel{k \rightarrow 0}{=} 3\left(\frac{k_{s}}{k}\right)^{2 \alpha},
$$

with $2 \alpha=2\left(n_{s}-4\right) / 3 \approx-2$, which in close analogy with the folded case leads to

$$
\begin{aligned}
& \Delta b_{\mathrm{I}, \mathrm{eql}}^{(\kappa)}(k) \\
& =6 f_{\mathrm{NL}}\left(\frac{\sigma_{\alpha s}}{\sigma_{0 s}}\right)^{2}\left[b_{1} \delta_{c}+2\left(\frac{\partial \ln \sigma_{\alpha s}}{\partial \ln \sigma_{0 s}}-1\right)\right] k^{-2 \alpha} \mathcal{M}_{s}^{-1}(k), \\
& \alpha=\left(n_{s}-4\right) / 3 \text {. }
\end{aligned}
$$

Our results agree with those of [14] (for the $k$-dependent $f_{\mathrm{NL}}$ ) and $[4,18]$ (for the folded and equilateral shapes) apart from a factor $\epsilon_{\alpha} /\left(b_{1} \delta_{c}\right)$, where

$$
\epsilon_{\alpha} \equiv b_{1} \delta_{c}+2\left(\frac{\partial \ln \sigma_{\alpha s}}{\partial \ln \sigma_{0 s}}-1\right) \text {. }
$$

This quantity clearly depends on the shape of primordial NG through the slope $\alpha$. The ratio $\epsilon_{\alpha} /\left(b_{1} \delta_{c}\right)$, which quantifies the deviation from the high-peak approximation, is shown in Fig. 2 for two $k$-dependent $f_{\mathrm{NL}}$ models with $n_{f}= \pm 0.6$ (dashed and dot-dashed curve), for the folded and orthogonal templates (long-dashed curve), and for the equilateral (dotted curve) bispectrum shapes. As can be seen, the ratio of the PBS to the high-peak prediction depends strongly on $b_{1}^{\mathrm{E}}$ and the value of $\alpha$. It is larger (smaller) than unity when $\alpha>0(\alpha<0)$. The suppression relative to the high-peak prediction is strongest for the equilateral bispectrum shape, for which $\alpha \approx-1$, but significant for all bispectrum shapes we considered. Clearly, this strong mass-dependence could be exploited to help constrain the shape of the primordial NG. As shown in [20], the results of N-body simulations match the predictions derived in this section well.

\section{PEAK-BACKGROUND SPLIT: CONDITIONAL MASS FUNCTION}

In this section, we consider the third derivation of the non-Gaussian bias based on the conditional halo mass function. This is essentially a peak-background split approach since we again consider the effect of adding a background perturbation $\delta_{l}$ of characteristic wavelength $R_{l} \gg R_{s}$ on the number density $\bar{n}_{h}$ of biased tracers. However, in contrast to the previous formulation, we consider a background density perturbation $\delta_{l}$ which is statistically correlated with small-scale density fluctuations. As shown in [19], such a peak-background split approach can be applied to obtain the scale-dependent bias factors of (Gaussian) density peaks at all orders. Here, we demonstrate that the implementation of [19] can be generalized to derive the non-Gaussian bias corrections.

In what follows, $\hat{\bar{n}}_{h}$ and $\hat{P}_{i}$ will denote non-Gaussian number densities and probability distributions, whereas $\bar{n}_{h}$ and $P_{i}$ will designate the Gaussian quantities. Since we will hereafter deal with the non-Gaussian density field, we shall revert to the notation of Sec. III and simply denote the latter as $\delta_{s}, \delta_{l}$ (and $\nu_{s}, \nu_{l}$ ). For simplicity, and since we are interested in the behavior on large scales, we shall ignore the peak constraint, which leads to corrections scaling as $k^{2}$ and higher powers. In other words, we will assume that, for Gaussian initial conditions, the number density of virialized objects $\bar{n}_{h}\left(\nu, R_{s}\right)$ identified on the scale $R_{s}$ follows a Press-Schechter mass function.

\section{A. Cumulants and conditional mass function}

Extending the derivation of the Press-Schechter mass function to the non-Gaussian case, we start from 


$$
\begin{aligned}
\hat{\bar{n}}_{h}\left(\nu, R_{s}\right) & =-2 \frac{\bar{\rho}}{M} \frac{d \hat{P}_{1}(>\nu, M)}{d M} \\
& =-2 \frac{\bar{\rho}}{M} \frac{d}{d M} \int_{\nu}^{\infty} d x \hat{P}_{1}\left(x, R_{s}\right),
\end{aligned}
$$

where $\hat{P}_{1}\left(\nu, R_{S}\right)$ is the probability that the linear density contrast of a Lagrangian region of mass $M \propto R_{s}^{3}$ equals $\delta_{c}=\nu \sigma_{0 s}$, and $\hat{P}_{1}(>\nu, M)$ is the probability that the same density contrast exceeds $\delta_{c}$. In this section, we shall use the parameter $\nu \equiv \delta_{c} / \sigma_{0 s}$ exclusively for the significance corresponding to the critical density with smoothing $R_{s}$. On the other hand, $\nu_{s} \equiv \delta_{s} / \sigma_{0 s}$ and $\nu_{l} \equiv \delta_{l} / \sigma_{0 l}$ stand for $N(0,1)$-distributed stochastic variables corresponding to density perturbations on small and large scales, respectively. As in Sec. III, we express the non-Gaussian joint probability density $P(\mathbf{y})$ for the $N$-dimensional vector of variables $\mathbf{y}$ in terms of the corresponding Gaussian probability density, by using the following general expansion:

$$
\begin{aligned}
\hat{P}(\mathbf{y})= & \exp \left[\sum_{m=3}^{\infty} \frac{(-1)^{m}}{m !} \sum_{\mu_{1} \cdots \mu_{m}}^{N}\left\langle y_{\mu_{1}} \cdots y_{\mu_{m}}\right\rangle_{c}\right. \\
& \left.\times \frac{\partial^{m}}{\partial y_{\mu_{1}} \cdots \partial y_{\mu_{m}}}\right] P(\mathbf{y}),
\end{aligned}
$$

where $\left\langle y_{\mu_{1}} \cdots y_{\mu_{m}}\right\rangle_{c}$ are connected cumulants and $P(\mathbf{y})$ is the multivariate Gaussian distribution characterized by the covariances $\left\langle y_{\mu_{1}} y_{\mu_{2}}\right\rangle$, e.g.,[62]. On inserting this expression into Eq. (90), the non-Gaussian mass function becomes

$$
\begin{aligned}
\hat{\bar{n}}_{h}\left(\nu, R_{s}\right)= & -\sqrt{\frac{2}{\pi}} \frac{\bar{\rho}}{M} \frac{d}{d M} \int_{\nu\left(R_{s}\right)}^{\infty} d x \\
& \times \exp \left[\sum_{m=3}^{\infty} \frac{(-1)^{m}}{m !}\left\langle\nu_{s}^{m}\right\rangle_{c} \frac{\partial^{m}}{\partial x^{m}}\right] e^{-x^{2} / 2} \\
\approx & \sqrt{\frac{2}{\pi}} \nu e^{-\nu^{2} / 2}\left(1+\sum_{m=3}^{\infty} \frac{\left\langle\nu_{s}^{m}\right\rangle_{c}}{m !} H_{m}(\nu)\right) \frac{\bar{\rho}}{M^{2}} \frac{d \ln \nu}{d \ln M} \\
& -\sqrt{\frac{2}{\pi}} \frac{\bar{\rho}}{M} \int_{\nu}^{\infty} d x e^{-x^{2} / 2} \frac{d}{d M}\left(1+\sum_{m=3}^{\infty} \frac{\left\langle\nu_{s}^{m}\right\rangle_{c}}{m !} H_{m}(x)\right) .
\end{aligned}
$$

In the second line, we have assumed that all the cumulants are much smaller than unity. This formula agrees with that obtained by [53] at first-order. Note that the excursion-set approach yields additional, albeit small corrections to the Press-Schechter expressions [54]. However, we will ignore them in what follows.

We now calculate the conditional mass function $\hat{\bar{n}}_{h}\left(\nu, R_{s} \mid \nu_{l}, R_{l}\right)$. By definition, the conditional probability for having a small-scale overdensity $\nu_{s}$ on scale $R_{s}$ given a large-scale overdensity $\nu_{l}$ on scale $R_{l}$ is

$$
\hat{P}\left(\nu_{s}, R_{s} \mid \nu_{l}, R_{l}\right)=\frac{\hat{P}_{2}\left(\nu_{s}, R_{s}, \nu_{l}, R_{l}\right)}{\hat{P}_{1}\left(\nu_{l}, R_{l}\right)} .
$$

The resulting conditional mass function thus is

$$
\begin{aligned}
\hat{\bar{n}}_{h}\left(\nu, R_{s} \mid \nu_{l}, R_{l}\right)= & -2 \frac{\bar{\rho}}{M} \frac{d}{d M} \int_{\nu}^{\infty} d \nu_{s} \hat{P}\left(\nu_{s}, R_{s} \mid \nu_{l}, R_{l}\right) \\
= & -2 \frac{\bar{\rho}}{M}\left[\hat{P}_{1}\left(\nu_{l}, R_{l}\right)\right]^{-1} \\
& \times \frac{d}{d M} \int_{\nu}^{\infty} d x \hat{P}_{2}\left(x, R_{s}, \nu_{l}, R_{l}\right) .
\end{aligned}
$$

The joint probability distribution $\hat{P}_{2}\left(\nu, R_{s}, \nu_{l}, R_{l}\right)$ is readily obtained from Eq. (91),

$$
\begin{aligned}
& \hat{P}_{2}\left(\nu, R_{s}, \nu_{l}, R_{l}\right) \\
& \approx\left(1+\sum_{N=3}^{\infty} \sum_{m=0}^{N} \frac{\left\langle\nu_{s}^{m} \nu_{l}^{N-m}\right\rangle_{c}}{m !(N-m) !} H_{m, N-m}\left(\nu, \nu_{l}, \epsilon\right)\right) \\
& \times \frac{f\left(\nu, \nu_{l}, \epsilon\right)}{2 \pi \sqrt{1-\epsilon^{2}}} .
\end{aligned}
$$

Here, the correlator stands for

$$
\left\langle\nu_{s}^{m} \nu_{l}^{N-m}\right\rangle_{c}=\sigma_{0 s}^{-m} \sigma_{0 l}^{N-m}\left\langle\delta_{s}^{m}(\boldsymbol{x}) \delta_{l}^{N-m}(\boldsymbol{x})\right\rangle_{c},
$$

where $\boldsymbol{x}$ is an arbitrary spatial location. The function $f\left(\nu, \nu_{l}, \epsilon\right)$ is the exponential piece of the Gaussian bivariate distribution, whereas $H_{m n}\left(\nu, \nu_{l}, \epsilon\right)$ are bivariate Hermite polynomials. They can be computed by taking derivatives of $f\left(\nu, \nu_{l}, \epsilon\right)$. Namely,

$$
\begin{aligned}
(-1)^{m+n} \frac{d^{m}}{d \nu^{m}} \frac{d^{n}}{d \nu_{l}^{n}} f\left(\nu, \nu_{l}, \epsilon\right) & =f\left(\nu, \nu_{l}, \epsilon\right) H_{m n}\left(\nu, \nu_{l}, \epsilon\right), \\
f\left(\nu, \nu_{l}, \epsilon\right) & \equiv \exp \left[-\frac{\nu^{2}+\nu_{l}^{2}-2 \epsilon \nu \nu_{l}}{2\left(1-\epsilon^{2}\right)}\right] .
\end{aligned}
$$

We define mixed spectral moments via

$$
\sigma_{n \times}^{2} \equiv \frac{1}{2 \pi^{2}} \int_{0}^{\infty} d k k^{2(n+1)} P_{\phi}(k) \mathcal{M}_{s}(k) \mathcal{M}_{l}(k),
$$

quantifying the cross-correlation between small and large scales (the $\times$ denotes the splitting of smoothing scales: one filter is of size $R_{s}$, the other of size $\left.R_{l}\right)$. Further, we define the quantity $\Sigma_{x}^{2}$ as

$$
\Sigma_{\times}^{2} \equiv \frac{1}{2 \pi^{2}} \int_{0}^{\infty} d k k^{2} P_{\phi}(k) \mathcal{M}_{s}(k) \mathcal{M}_{l}(k) \mathcal{S}\left(k, R_{s}, R_{l}\right)
$$

where the form factor $\mathcal{S}$ generally is a function of $k, R_{s}$ and $R_{l}$. This definition is broad enough to describe all the spectral moments and the cumulants of the density field. For instance, setting $\mathcal{S}\left(k, R_{s}, R_{l}\right)=k^{2}$ yields $\Sigma_{\times}^{2}=\sigma_{1 \times}^{2}$. In the following, we will use the following kernel for $\Sigma_{x}^{2}$ :

$$
\mathcal{S}\left(k, R_{s}\right) \equiv 4 \sigma_{0 s}^{2} \mathcal{F}_{s}^{(N)}(k, X) \mathcal{M}_{s}^{-1}(k) .
$$


Inserting the expression for the form factor Eq. (42), we see that $\Sigma_{\times}^{2}$ becomes

$$
\Sigma_{\times}^{2}=\sigma_{0 s}^{N-1} \sigma_{0 l}\left\langle\nu_{s}^{N-1} \nu_{l}\right\rangle_{c},
$$

i.e., a mixed $N$-th order moment of the density field induced by the primordial $N$-point function.

\section{B. Relative overabundance of rare objects}

The non-Gaussian corrections to the $N$-th order Gaussian bias parameters $b_{N}$ can be calculated by expanding the relative overabundance of biased tracers $\hat{\bar{n}}_{h}\left(\nu, R_{s} \mid \nu_{l}, R_{l}\right) / \hat{\bar{n}}_{h}\left(\nu, R_{s}\right)-1$ at order $\delta_{l}^{N}$. However, throughout the remainder of this section, we will consider only the correction to the linear bias. Taking the ratio of the conditional mass function to the universal one yields

$$
\begin{aligned}
\delta_{h}\left(\delta_{l}\right) & \equiv \frac{\hat{\bar{n}}_{h}\left(\nu, R_{s} \mid \nu_{l}, R_{l}\right)}{\hat{\bar{n}}_{h}\left(\nu, R_{s}\right)}-1 \\
& =\frac{\frac{d}{d M} \int_{\nu}^{\infty} d x \hat{P}_{2}\left(x, R_{s}, \nu_{l}, R_{l}\right)}{\hat{P}_{1}\left(\nu_{l}, R_{l}\right) \frac{d}{d M} \int_{\nu}^{\infty} d x \hat{P}_{1}\left(x, R_{s}\right)}-1 .
\end{aligned}
$$

Now comes a crucial step in the calculation. As $R_{l}$ increases, the ratio $\left\langle\nu_{s}^{m} \nu_{l}^{N-m}\right\rangle / \sigma_{0 l}^{2}$ (which is the analog of $\sigma_{n \times}^{2} / \sigma_{0 l}^{2}$ in the calculation of the peak bias factors) remains finite only if the corresponding form factor $\mathcal{S}\left(k, R_{s}, R_{l}\right)$ does not depend on $R_{l}$ (again, this applies when expanding to linear order in $\nu_{l}$ ). This implies that, in Eq. (102), only the terms involving the cumulants $\left\langle\nu_{s}^{N}\right\rangle_{c}$ or $\left\langle\nu_{s}^{N-1} \nu_{l}\right\rangle_{c}$ will survive. Therefore, upon taking the limit $R_{l} \rightarrow \infty$, we arrive at

$$
\delta_{h}\left(\delta_{l}\right)=\frac{\frac{d}{d M} \int_{\nu}^{\infty} d x\left\{1+\sum_{N=3}^{\infty} \frac{1}{N !}\left[\left\langle\nu_{s}^{N}\right\rangle_{c} H_{N, 0}\left(x, \nu_{l}, \epsilon\right)+N\left\langle\nu_{s}^{N-1} \nu_{l}\right\rangle_{c} H_{N-1,1}\left(x, \nu_{l}, \epsilon\right)\right]\right\} \frac{\exp \left[-\frac{\left(x-\epsilon \nu^{2}\right)^{2}}{2\left(1-\epsilon^{2}\right)^{2}}\right]}{\sqrt{1-\epsilon^{2}}}}{\frac{d}{d M} \int_{\nu}^{\infty} d x\left[1+\sum_{N=3}^{\infty} \frac{\left\langle\nu_{s}^{N}\right\rangle_{c}}{N !} H_{N}(x)\right] e^{-x^{2} / 2}}-1 .
$$

In order to calculate the non-Gaussian contribution to $b_{1}$, it is sufficient to expand the right-hand side of Eq. (103) at order $\delta_{l}$. The first term appearing in the square brackets can be re-expressed as

$$
\frac{1}{N !}\left\langle\nu_{s}^{N}\right\rangle_{c} H_{N, 0}\left(x, \nu_{l}, \epsilon\right)=\frac{1}{N !}\left\langle\nu_{s}^{N}\right\rangle_{c}\left(1-\epsilon^{2}\right)^{-N / 2} H_{N}\left(\frac{x-\epsilon \nu_{l}}{\sqrt{1-\epsilon^{2}}}\right) \approx \frac{1}{N !}\left\langle\nu_{s}^{N}\right\rangle_{c}\left[H_{N}(x)-N \epsilon \nu_{l} H_{N-1}(x)\right]+\mathcal{O}\left(\nu_{l}^{2}\right) .
$$

In the second line, we successively set $\epsilon \rightarrow 0$ (we can ignore terms involving $\epsilon^{2}$ ) and employed the relation $H_{N}^{\prime}(x)=N H_{N-1}(x)$ to expand the result at first order in $\nu_{l}$. To simplify the second term in the curly brackets of Eq. (103), we use the fact that $f\left(\nu, \nu_{l}, \epsilon\right)$ in Eq. (97) satisfies the following identity

$$
\left[\frac{\partial}{\partial \nu_{l}}+\epsilon \frac{\partial}{\partial x}\right] f\left(x, \nu_{l}, \epsilon\right)=-\nu_{l} f\left(x, \nu_{l}, \epsilon\right)
$$

Therefore,

$$
\begin{aligned}
H_{N-1,1}\left(x, \nu_{l}, \epsilon\right) & \equiv \frac{(-1)^{N}}{f\left(x, \nu_{l}, \epsilon\right)} \frac{\partial^{N-1}}{\partial x^{N-1}} \frac{\partial}{\partial \nu_{l}} f\left(x, \nu_{l}, \epsilon\right) \\
& =-\epsilon \frac{(-1)^{N}}{f\left(x, \nu_{l}, \epsilon\right)} \frac{\partial^{N}}{\partial x^{N}} f\left(x, \nu_{l}, \epsilon\right)+\nu_{l} \frac{(-1)^{N-1}}{f\left(x, \nu_{l}, \epsilon\right)} \frac{\partial^{N-1}}{\partial x^{N-1}} f\left(x, \nu_{l}, \epsilon\right) \\
& =-\frac{\epsilon}{\left(1-\epsilon^{2}\right)^{N / 2}} H_{N}\left(\frac{x-\epsilon \nu_{l}}{\sqrt{1-\epsilon^{2}}}\right)+\frac{\nu_{l}}{\left(1-\epsilon^{2}\right)^{(N-1) / 2}} H_{N-1}\left(\frac{x-\epsilon \nu_{l}}{\sqrt{1-\epsilon^{2}}}\right) \approx \nu_{l} H_{N-1}(x) .
\end{aligned}
$$

We thus obtain

$$
\begin{aligned}
& \frac{1}{(N-1) !}\left\langle\nu_{s}^{N-1} \nu_{l}\right\rangle_{c} H_{N-1,1}\left(x, \nu_{l}, \epsilon\right) \\
& \quad \approx \frac{\nu_{l}}{(N-1) !}\left\langle\nu_{s}^{N-1} \nu_{l}\right\rangle_{c} H_{N-1}(x)+\mathcal{O}\left(\nu_{l}^{2}\right) .
\end{aligned}
$$

On expanding the numerator of Eq. (103) at first order in $\nu_{l}$, we can isolate the Gaussian contribution, which is

$$
\frac{\frac{d}{d M} \int_{\nu}^{\infty} d x \epsilon \nu_{l} x e^{-x^{2} / 2}}{\frac{d}{d M} \int_{\nu}^{\infty} d x e^{-x^{2} / 2}}=\epsilon \nu_{l}\left(\nu-\frac{1}{\nu}\right)=\left(\frac{\sigma_{0 \times}^{2}}{\sigma_{0 l}^{2}}\right) b_{1} \delta_{l},
$$

in agreement with the linear PBS bias for the PressSchechter mass function derived in Sec. III. We now retain all the terms linear in the higher-order cumulants $(N \geq 3)$ in the linear expansion of Eqs. (92) and (95) and obtain 


$$
\begin{aligned}
\delta_{h}\left(\delta_{l}\right) \approx & \left(\frac{\sigma_{0 x}^{2}}{\sigma_{0 l}^{2}}\right) b_{1} \delta_{l}+\left(\frac{\sigma_{0 x}^{2}}{\sigma_{0 l}^{2}}\right) b_{1} e^{\nu^{2} / 2}\left[\sum_{N=3}^{\infty} \frac{d}{d M} \int_{\nu}^{\infty} d x \frac{\left\langle\nu_{s}^{N}\right\rangle_{c}}{N !} H_{N}(x) e^{-x^{2} / 2}\right] \frac{d M}{d \nu} \delta_{l} \\
& -e^{\nu^{2} / 2}\left[\sum_{N=3}^{\infty} \frac{d}{d M} \int_{\nu}^{\infty} d x \epsilon \nu_{l} \frac{\left\langle\nu_{s}^{N}\right\rangle_{c}}{N !} H_{N}(x) x e^{-x^{2} / 2}\right] \frac{d M}{d \nu} \\
& -e^{\nu^{2} / 2}\left\{\sum_{N=3}^{\infty} \frac{d}{d M} \int_{\nu}^{\infty} d x\left[-\frac{\left\langle\nu_{s}^{N}\right\rangle_{c}}{(N-1) !} \epsilon \nu_{l}+\frac{\left\langle\nu_{s}^{N-1} \nu_{l}\right\rangle_{c}}{(N-1) !} \nu_{l}\right] H_{N-1}(x) e^{-x^{2} / 2}\right\} \frac{d M}{d \nu} .
\end{aligned}
$$

Using the generating function $\exp \left(x t-t^{2} / 2\right)=\sum_{N} H_{N}(x) t^{N} / N$ !, we can easily evaluate the integrals over the Hermite polynomials. In particular, we find for $N \geq 2$ :

$$
\int_{\nu}^{\infty} d x x H_{N}(x) e^{-x^{2} / 2}=\left[\nu H_{N-1}(\nu)+H_{N-2}(\nu)\right] e^{-\nu^{2} / 2} .
$$

On inserting this expression into Eq. (109), taking the derivative with respect to $M$ and employing the recurrence relation $H_{N+1}(x)=x H_{N}(x)-N H_{N-1}(x)$, the conditional overabundance of halos simplifies to

$$
\begin{aligned}
\delta_{h}\left(\delta_{l}\right) \approx & \left(\frac{\sigma_{0 \times}^{2}}{\sigma_{0 l}^{2}}\right) b_{1} \delta_{l}-\left(\frac{\sigma_{0 \times}^{2}}{\sigma_{0 l}^{2}}\right) b_{1} \sum_{N=3}^{\infty} \frac{1}{N !}\left[\sigma_{0 s}^{2} \delta_{c}^{-1}\left\langle\nu_{s}^{N}\right\rangle_{c}^{\prime} H_{N-1}(\nu)+\left\langle\nu_{s}^{N}\right\rangle_{c} H_{N}(\nu)\right] \delta_{l} \\
& -\left(\frac{\sigma_{0 \times}^{2}}{\sigma_{0 l}^{2}}\right) \sum_{N=3}^{\infty} \frac{1}{N !}\left[-\sigma_{0 s}^{2} \delta_{c}^{-1}\left\langle\nu_{s}^{N}\right\rangle_{c}^{\prime} H_{N}(\nu)+\left\langle\nu_{s}^{N}\right\rangle_{c}\left(-H_{N+1}(\nu)+\frac{H_{N}(\nu)}{\nu}\right)\right] \frac{\delta_{l}}{\sigma_{0 s}} \\
& +\sum_{N=3}^{\infty} \frac{1}{(N-1) !}\left[\sigma_{0 s}^{2} \delta_{c}^{-1}\left\langle\nu_{s}^{N-1} \nu_{l}\right\rangle_{c}^{\prime} H_{N-2}(\nu)+\left\langle\nu_{s}^{N-1} \nu_{l}\right\rangle_{c} H_{N-1}(\nu)\right] \frac{\delta_{l}}{\sigma_{0 l}}
\end{aligned}
$$

where a primed variable $X^{\prime}$ now designates $\partial X / \partial \sigma_{0 s}$ (we have used the fact that $d \nu=-\delta_{c} d \sigma_{0 s} / \sigma_{0 s}^{2}$ ).

\section{Non-Gaussian bias corrections}

In order to calculate the non-Gaussian bias corrections, we have to compute the derivative of the $N$-point cumulants $\left\langle\nu_{s}^{N}\right\rangle_{c}=\sigma_{0 s}^{N-2} S_{s}^{(N)}$ and $\left\langle\nu_{s}^{N-1} \nu_{l}\right\rangle_{c} \equiv \Sigma_{\times}^{2} /\left(\sigma_{0 s}^{N-1} \sigma_{0 l}\right)$ with respect to $\sigma_{0 s}$. These are

$$
\begin{gathered}
\left\langle\nu_{s}^{N}\right\rangle_{c}^{\prime}=\sigma_{0 s}^{N-3} S_{s}^{(N)}\left[(N-2)+\frac{\partial \ln S_{s}^{(N)}}{\partial \ln \sigma_{0 s}}\right], \\
\left\langle\nu_{s}^{N-1} \nu_{l}\right\rangle_{c}^{\prime}=\frac{1}{\sigma_{0 s}^{N} \sigma_{0 l}}\left[\sigma_{0 s} \frac{\partial\left(\Sigma_{\times}^{2}\right)}{\partial \sigma_{0 s}}-(N-1) \Sigma_{\times}^{2}\right] .
\end{gathered}
$$

Replacing the Hermite polynomials with the Gaussian peak-background split biases inferred from the Press-Schechter multiplicity function [Eq. (28)],

$$
b_{N}(\nu)=\frac{1}{\sigma_{0 s}^{N}} \frac{H_{N+1}(\nu)}{\nu},
$$

the conditional overabundance of halos can be recast into

$$
\begin{aligned}
\delta_{h}\left(\delta_{l}\right) \approx & \left(\frac{\sigma_{0 \times}^{2}}{\sigma_{0 l}^{2}}\right) b_{1} \delta_{l}-\left(\frac{\sigma_{0 \times}^{2}}{\sigma_{0 l}^{2}}\right) \sum_{N=3}^{\infty} \frac{S_{s}^{(N)}}{N !}\left[(N-2)+\frac{\partial \ln S_{s}^{(N)}}{\partial \ln \sigma_{0 s}}\right] \sigma_{0 s}^{2(N-2)}\left(b_{1} b_{N-2}-b_{N-1}\right) \delta_{l} \\
& -\left(\frac{\sigma_{0 \times}^{2}}{\sigma_{0 l}^{2}}\right) \sum_{N=3}^{\infty} \frac{S_{s}^{(N)}}{N !} \sigma_{0 s}^{2(N-2)}\left(\delta_{c} b_{1} b_{N-1}+b_{N-1}-\delta_{c} b_{N}\right) \delta_{l} \\
& +\sum_{N=3}^{\infty} \frac{\sigma_{0 s}^{-2}}{(N-1) !}\left[\left(\frac{\left(\sum_{\times}^{2}\right)^{\prime}}{\sigma_{0 l}^{2}}\right) \sigma_{0 s} b_{N-3}-\left(\frac{\sum_{\times}^{2}}{\sigma_{0 l}^{2}}\right)(N-1) b_{N-3}+\left(\frac{\Sigma_{\times}^{2}}{\sigma_{0 l}^{2}}\right) \delta_{c} b_{N-2}\right] \delta_{l} .
\end{aligned}
$$

We can now read off the scale-independent correction $\Delta b_{I}^{(l)}$ (involving the terms proportional to $\sigma_{0 \times}^{2} / \sigma_{0 l}^{2}$ ) and a scale-dependent correction $\Delta \mathrm{b}_{\mathrm{I}}^{(\kappa)}$ (involving the terms $\Sigma_{\times}^{2} / \sigma_{0 l}^{2}$ and $\left.\left(\Sigma_{\times}^{2}\right)^{\prime} / \sigma_{0 l}^{2}\right)$ to the first-order Gaussian bias $b_{1}$. The non-Gaussian bias contribution thus is $\Delta \mathrm{b}_{\mathrm{I}}=\Delta \mathrm{b}_{\mathrm{I}}^{(\iota)}+\Delta \mathrm{b}_{\mathrm{I}}^{(\kappa)}$. After some manipulation, the scale-independent non-Gaussian bias correction reads 


$$
\begin{aligned}
\Delta \mathrm{b}_{\mathrm{I}}^{(\iota)}\left(R_{s}, X\right) & \\
= & -\frac{S_{s}^{(N)}(X)}{N !} \sigma_{0 s}^{2(N-2)}\left\{\left[(N-2)+\frac{\partial \ln S_{s}^{(N)}(X)}{\partial \ln \sigma_{0 s}}\right]\right. \\
& \left.\times\left(b_{1} b_{N-2}-b_{N-1}\right)-\left(\delta_{c} b_{1} b_{N-1}+b_{N-1}-\delta_{c} b_{N}\right)\right\},
\end{aligned}
$$

where $X$ is again a vector of variables describing the amplitude and shape of the primordial $N$-point function.
In order to write down an explicit expression for the scale-dependent, non-Gaussian bias correction, we use the definition of the kernel $\mathcal{S}\left(k, R_{s}\right)$ [Eq. (100)], yielding

$$
\Sigma_{\times}^{2}=4 \sigma_{0 s}^{2} \int \frac{d^{3} k}{(2 \pi)^{3}} P_{\phi}(k) \mathcal{M}_{l}(k) \mathcal{F}_{s}^{(N)}(k, X) .
$$

Then, by definition of the linear halo bias, $\delta_{h}(\mathbf{k})=$ $b(k) \delta_{s}(\mathbf{k})$, correlating the last line of Eq. (98) with $\delta_{l}$ yields

$$
\begin{aligned}
\int \frac{d^{3} k}{(2 \pi)^{3}} \Delta b_{I}^{(\kappa)} P_{\phi}(k) \mathcal{M}_{s}(k) \mathcal{M}_{l}(k)= & \sum_{N=3}^{\infty} \frac{\sigma_{0 s}^{-2}}{(N-1) !}\left\{\left(\Sigma_{\times}^{2}\right)^{\prime} \sigma_{0 s} b_{N-3}-\Sigma_{\times}^{2}\left[(N-1) b_{N-3}-\delta_{c} b_{N-2}\right]\right\} \\
= & \int \frac{d^{3} k}{(2 \pi)^{3}} P_{\phi}(k) \mathcal{M}_{l}(k) \sum_{N=3}^{\infty} \frac{4}{(N-1) !} \\
& \times\left\{\frac{d\left(\sigma_{0 s}^{2} \mathcal{F}_{s}^{(N)}\right)}{d \sigma_{0 s}} \sigma_{0 s}^{-1} b_{N-3}-\mathcal{F}_{s}^{(N)}\left[(N-1) b_{N-3}-\delta_{c} b_{N-2}\right]\right\}
\end{aligned}
$$

from which we can read off the scale-dependent non-Gaussian bias correction as

$$
\Delta \mathrm{b}_{\mathrm{I}}^{(\kappa)}\left(k, R_{s}, X\right)=\frac{4}{(N-1) !}\left\{b_{N-2} \delta_{c}+b_{N-3}\left[3-N+\frac{\partial \ln \mathcal{F}_{s}^{(N)}(k, X)}{\partial \ln \sigma_{0 s}}\right]\right\} \mathcal{F}_{s}^{(N)}(k, X) \mathcal{M}_{s}^{-1}(k) .
$$

This is the main result of this section. In the high-peak limit, $b_{N-2} \gg b_{N-3}$ and the first term in the curly bracket dominates. Therefore, we exactly recover the results of [5,13,14,31] for the constant $f_{\mathrm{NL}}$, constant $g_{\mathrm{NL}}$, folded shape and $k$-dependent $f_{\mathrm{NL}}$, respectively. The second term in the curly brackets arises owing to the massdependence of the reduced cumulants $S_{s}^{(N)}$. As we will see shortly, this term agrees with the correction derived in Sec. IV in the limit $k \rightarrow 0$.

Note that [63] also employed the bivariate Edgeworth expansion to explore the effect of a local primordial trispectrum on the (configuration space) bias of tracers. However, they did not derive any explicit expression for the non-Gaussian bias.

\section{Comparison of the PBS approaches}

Interestingly, if we ignore the mass-dependence of the cumulants, then the $k$-dependence of Eq. (118) is exactly the same as that predicted by the correlation of thresholded regions (see Sec. III). This follows from expanding the non-Gaussian density field in cumulants, which is also done in the expansion of the correlation function of thresholded regions. By contrast, our first formulation of the peak-background split (see Sec. IV) leads to a different $k$-dependence on smaller scales. This difference arises because we have assumed that the long-wavelength perturbation is constant over some intermediate scale $R$ over which the halo abundance is averaged. This is a sensible assumption as long as the scale " $L$ " over which we measure the clustering of halos is much larger than the scales that contribute to $\sigma_{0 s}$. Then, the kernel $\omega$ in Eq. (63) is indeed evaluated in the squeezed limit, $k_{s} \gg k$, and both PBS formulations agree exactly. To see this explicitly, we write Eq. (118) for the cases of $N=3$ and $N=4$ :

$$
\begin{aligned}
& \Delta \mathrm{b}_{\mathrm{I}}^{(\kappa)}\left(k, R_{s}, N=3\right) \\
& \quad=2\left\{b_{1} \delta_{c}+\frac{\partial \ln \mathcal{F}_{s}^{(3)}(k)}{\partial \ln \sigma_{0 s}}\right\} \mathcal{F}_{s}^{(3)}(k) \mathcal{M}_{s}^{-1}(k)
\end{aligned}
$$

$$
\begin{aligned}
& \Delta \mathrm{b}_{\mathrm{I}}^{(\kappa)}\left(k, R_{s}, N=4\right) \\
& \quad=\frac{4}{6}\left\{b_{2} \delta_{c}+b_{1}\left[1+\frac{\partial \ln \mathcal{F}_{s}^{(4)}(k)}{\partial \ln \sigma_{0 s}}\right]\right\} \mathcal{F}_{s}^{(4)}(k) \mathcal{M}_{s}^{-1}(k) .
\end{aligned}
$$

In the large-scale limit, we can use the same approximations made in Sec. IV, i.e., assume that $k$ is much smaller than the scales which contribute significantly to the integrand in Eq. (42). On inserting the definition of the kernels $\omega^{(2)}, \omega^{(3)}$ [Eqs. (58) and (59)], we obtain

$$
\begin{gathered}
\mathcal{F}_{s}^{(3)}(k) \stackrel{k \rightarrow 0}{=} f_{\mathrm{NL}} \frac{\sigma_{\omega s}^{2}(k)}{\sigma_{0 s}^{2}} \\
\mathcal{F}_{s}^{(4)}(k) \stackrel{k \rightarrow 0}{=} \frac{3}{4} g_{\mathrm{NL}} \sigma_{0 s}^{2} S_{\omega s}^{(3)}(k) .
\end{gathered}
$$

Substituting these expressions into Eqs. (119) and (120), we eventually recover Eq. (78) in Sec. IV. 


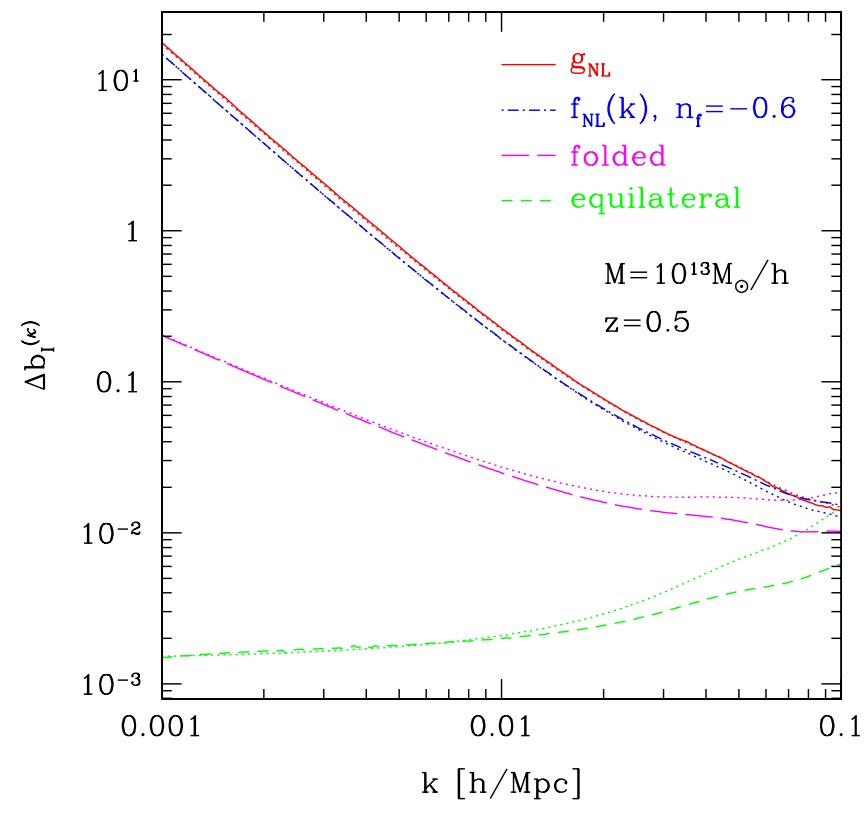

FIG. 3 (color online). A comparison between the nonGaussian scale-dependent bias correction Eq. (118) and its low- $k$ limit Eq. (78) for some of the bispectrum shapes and the local trispectrum considered in this work. In all cases, a dotted curve represents the low- $k$ limit. Results are shown as a function of $k$ for halos of mass $M=5.3 \times 10^{13} \mathrm{M}_{\odot} / \mathrm{h}$ at $z=0.5$, assuming $f_{\mathrm{NL}}=100$ (for the bispectra) and $g_{\mathrm{NL}}=10^{6}$ (for the local trispectrum).

On smaller scales $k \gtrsim 0.02 \mathrm{~h} / \mathrm{Mpc}$ around which the matter power spectrum peaks, the separation of scales " $L$ " and " $S$ " is no longer accurate and the predictions of Eq. (118) diverge from the $k \rightarrow 0$ limit. In Fig. 3, the exact $k$-dependence of the non-Gaussian bias correction predicted by the correlated PBS approach, Eq. (118), is compared to that predicted by the low- $k$ expression, Eq. (78). We can see that the latter is accurate to a few percent at wave number $k \lessgtr 0.01 \mathrm{~h} \mathrm{Mpc}^{-1}$. Only for the folded and equilateral shape does the low- $k$ expression yield a noticeably larger non-Gaussian bias correction on scales $k \gtrsim 0.01 \mathrm{~h} \mathrm{Mpc}^{-1}$. This is also true for the orthogonal template (not shown in the figure since it is essentially equal to the folded case). The exact difference, however, depends somewhat on halo mass and redshift. A quantitative comparison of the scale-dependent bias predicted by the uncorrelated PBS approach with that obtained from the statistics of thresholded regions can also be found in [18] (note however that the new term derived in this work is not included there).

Finally, while in the limit $k \rightarrow 0$ Eq. (118) reproduces the well-known result for the local scale-independent $f_{\mathrm{NL}}$ model [3,5], at finite $k$, this expression receives a negative correction from the second term proportional to $\partial \ln \mathcal{F}_{s}^{(3)} / \partial \ln \sigma_{0 s}$ that increases with wave number. At $k=$ $0.05 \mathrm{hMpc}^{-1}$ for instance, the suppression is $\sim 1 \%$ and $\sim 4 \%$ for biased tracers with $b_{1}^{\mathrm{E}} \sim 2$ and 3.5, respectively.

\section{PEAK-BACKGROUND SPLIT VS THRESHOLDING}

We now compare our final result Eq. (118), with the result from thresholding in the high-peak limit,

$$
\Delta \mathrm{b}_{\mathrm{I}}^{(\mathrm{hp})}(k)=\frac{4 b_{N-1}}{(N-1) !} \sigma_{0 s}^{2} \frac{\mathcal{F}_{s}^{(N)}(k)}{\mathcal{M}_{s}(k)},
$$

obtained from Eq. (43) by replacing $c_{N}$ with $b_{N}$. We see two important differences. Firstly, in the thresholding approach (which is equivalent to local biasing), the correction to the halo power spectrum induced by a primordial $N$-point function is proportional to $b_{N-1}$. In the PBS approach on the other hand, the correction comes in through the dependence of the halo mass function on the $(N-1)$-th moment $S_{s}^{(N-1)}$ of the small-scale density field. The latter is proportional to $b_{N-2}$ when the Edgeworth approximation method is applied to the halo mass function. The simulation results for all types of primordial NG simulated so far clearly follow the dependence on $b_{N-2}$ rather than $b_{N-1}$, thus favoring the interpretation provided by the PBS approach.

Secondly, the term proportional to $\partial \ln \mathcal{F}_{s}^{(3)} / \partial \ln \sigma_{0 s}$ in the PBS prediction [Eq. (118)] is absent in the thresholding approach. In [20], we show that the inclusion of this term yields a good match to the simulated halo bias in nonGaussian models beyond the simplest, local quadratic NG with scale-independent $f_{\mathrm{NL}}$. In the thresholding approach on the other hand, we associate the correlation of regions above a threshold $\delta_{c}(z)$ in the linear density field smoothed at a fixed scale $R_{s}$ with that of halos above a mass threshold $M\left(R_{s}\right)$ at redshift $z$. However, halos spanning some mass interval should be identified with Lagrangian regions spanning a range of smoothing scales. Consequently, the abundance of halos in a mass interval $[M, M+d M]$ not only depends on the cumulants $S_{s}^{(N-1)}$ of the density field smoothed on scale $R_{S}$, but also on the variation of these cumulants with $R_{s}$ (parameterized through $\left.\partial \ln S_{s}^{(N-1)} / \partial \ln \sigma_{0 s}\right)$.

An alternative way of seeing this is to describe the abundance of halos in a non-Gaussian density field through an effective significance $\hat{\nu}\left(\nu, S_{s}^{(m)}\right)$, which is defined upon requiring

$$
\hat{\bar{n}}_{h}=\frac{\bar{\rho}}{M^{2}} f_{\mathrm{NG}}(\nu) \frac{d \ln \nu}{d \ln M}=\frac{\bar{\rho}}{M^{2}} f_{\mathrm{G}}(\hat{\nu}) \frac{d \ln \hat{\nu}}{d \ln M} .
$$

In the case of a Press-Schechter mass function [Eq. (92)], $\hat{\nu}$ is given by

$$
\hat{\nu}=\nu\left(1-\sum_{m=3}^{\infty} \frac{1}{m !} S_{s}^{(m)} \sigma_{0 s}^{2(m-2)} b_{m-2}\right)
$$

The Jacobian $d \ln \hat{\nu} / d \ln M$ in Eq. (124) involves $\partial \ln S_{s}^{(m)} / \partial \ln \nu$, showing that $\hat{\bar{n}}_{h}$ depends on the scaledependence of the cumulants. 


\section{CONCLUSION}

We have carefully reexamined the derivation of the effect of primordial NG on the large-scale clustering of tracers (such as galaxies and clusters) beyond the local $f_{\mathrm{NL}}$ model, using the statistics of thresholded regions as well as two formulations of the peak-background split (PBS). We have shown that the thresholding approach is equivalent to local biasing, when considering the leading order contributions from NG. This approach predicts the same scaledependence as the PBS approach in the limit $k \rightarrow 0$, in agreement with the findings of [18]. However, unless we consider the high-peak limit (which is never attained by real tracers), PBS and thresholding predict different amplitudes for the non-Gaussian contribution to the linear bias.

We have presented two complementary peakbackground split derivations of the effect of NG. In the first approach, the separation of scales is invoked to split the Gaussian density field into uncorrelated short- and longwavelength perturbations. This allows us to isolate the mode-coupling effect responsible for the scale-dependent bias induced by NG. In the second approach, the separation of scales is invoked to expand the ratio of the unconditional to conditional mass function in terms of large-scale perturbations in the non-Gaussian density field. Notice that no assumption of separation of scales is made in the thresholding approach, where biasing is a function of the local density only. While in the second PBS approach we have restricted ourselves to the case of a Press-Schechter mass function, we have nonetheless been able to identify the nonGaussian bias correction to the linear bias. Both PBS approaches predict exactly the same correction in the limit $k \rightarrow 0$ (once the Press-Schechter expressions for the Gaussian biases are identified with $b_{N}$ ). While they depart at higher wavenumbers ( $k \geq 0.02 \mathrm{~h} / \mathrm{Mpc})$, this deviation is not very significant for the local or folded type of NG where the non-Gaussian bias correction is strongly suppressed at small scales.

In both approaches, we uncover a new term depending on the scale-dependence of the small-scale moments of the density field induced by NG. Physically, this term is induced by the mapping from local significance $\nu=\delta_{c} / \sigma_{0 s}$ to mass $M$ : a scale-dependent modulation of $\sigma_{0 s}$ changes the interval $d \nu$ corresponding to a fixed mass interval $d M$. This correction to the high-peak expression of the linear
non-Gaussian bias has not been pointed out in any previous work. It can be very large for all the models considered here, except for the local bispectrum with constant (i.e., $k$-independent) $f_{\mathrm{NL}}$. Moreover, we have found very good overall agreement between the PBS predictions and the simulated non-Gaussian halo bias [13-15] for the local $g_{\mathrm{NL}} \phi^{3}$ model, the local $f_{\mathrm{NL}} \phi^{2}$ model with $k$-dependent $f_{\mathrm{NL}}$, and the orthogonal bispectrum. This comparison is detailed in a companion Letter to this paper [20]. Consequently, the simulation results rule out thresholding, and more generally local biasing, as a viable approach to predicting the impact of primordial NG on halo clustering. These new accurate predictions can be combined with optimal weighting schemes [64-69] in order to extract information on the scale-dependent bias from numerical simulations and forthcoming galaxy surveys.

In order to further test the PBS approach with numerical simulations, it will be important to take into account the scale-independent correction $\Delta \mathrm{b}_{\mathrm{I}}^{(\iota)}$ induced by NG through its impact on the abundance of halos. In the case of local cubic NG, it will also be necessary to measure the Gaussian second-order bias factor $b_{2}$ directly from the simulations, as the effect on the linear bias scales with $b_{2}$.

Finally, a natural generalization of the conditional mass function approach discussed in Sec. V is a derivation of the non-Gaussian bias factors within the excursion-set formalism, for generic moving barriers and non-Gaussian initial conditions $[54,55,70,71]$. We leave these issues for more detailed future treatments.

\section{ACKNOWLEDGMENTS}

We are grateful to Tobias Baldauf, Olivier Doré, Chris Hirata, Marc Kamionkowski, Eichiiro Komatsu, Román Scoccimárro, Emiliano Sefusatti, Leonardo Senatore, Ravi Sheth for many fruitful discussions, and to Sirichai Chongchitnan and Ravi Sheth for comments on an early version of this manuscript. VD wishes to thank Theoretical Astrophysics at Caltech and the Center for Cosmological Physics at Berkeley for hospitality during the completion of parts of this work. DJ and FS are supported by the Gordon and Betty Moore Foundation at Caltech. VD is supported by the Swiss National Science Foundation under Contract No. 200021-116696/1 and by FK UZH 57184001.
[1] R. Scoccimarro, E. Sefusatti, and M. Zaldarriaga, Phys. Rev. D 69, 103513 (2004).

[2] E. Sefusatti, M. Crocce, S. Pueblas, and R. Scoccimarro, Phys. Rev. D 74, 023522 (2006).

[3] N. Dalal, O. Doré, D. Huterer, and A. Shirokov, Phys. Rev. D77, 123514 (2008).
[4] V. Desjacques and U. Seljak, arXiv:1006.4763.

[5] S. Matarrese and L. Verde, Astrophys. J. Lett. 677, L77 (2008).

[6] A. Slosar, C. Hirata, U. Seljak, S. Ho, and N. Padmanabhan, J. Cosmol. Astropart. Phys. 8 (2008) 031. 
[7] N. Afshordi and A. J. Tolley, Phys. Rev. D 78, 123507 (2008).

[8] V. Desjacques, U. Seljak, and I. T. Iliev, Mon. Not. R. Astron. Soc. 396, 85 (2009).

[9] A. Pillepich, C. Porciani, and O. Hahn, Mon. Not. R. Astron. Soc. 402, 191 (2010).

[10] M. Grossi, L. Verde, C. Carbone, K. Dolag, E. Branchini, F. Iannuzzi, S. Matarrese, and L. Moscardini, Mon. Not. R. Astron. Soc. 398, 321 (2009).

[11] T. Nishimichi, A. Taruya, K. Koyama, and C. Sabiu, J. Cosmol. Astropart. Phys. 7 (2010) 002.

[12] K. M. Smith and M. LoVerde, arXiv:1010.0055.

[13] V. Desjacques and U. Seljak, Phys. Rev. D 81, 023006 (2010).

[14] S. Shandera, N. Dalal, and D. Huterer, J. Cosmol. Astropart. Phys. 3 (2011) 017.

[15] C. Wagner and L. Verde, arXiv:1102.3229.

[16] B. Grinstein and M. B. Wise, Astrophys. J. 310, 19 (1986).

[17] S. Matarrese, F. Lucchin, and S. A. Bonometto, Astrophys. J. Lett. 310, L21 (1986).

[18] F. Schmidt and M. Kamionkowski, Phys. Rev. D 82, 103002 (2010).

[19] V. Desjacques, M. Crocce, R. Scoccimarro, and R. K. Sheth, Phys. Rev. D 82, 103529 (2010).

[20] V. Desjacques, D. Jeong, and F. Schmidt, arXiv:1105.3476 [Phys. Rev. Lett. (to be published).

[21] D. S. Salopek and J.R. Bond, Phys. Rev. D 42, 3936 (1990).

[22] A. Gangui, F. Lucchin, S. Matarrese, and S. Mollerach, Astrophys. J. 430, 447 (1994).

[23] L. Verde, L. Wang, A. F. Heavens, and M. Kamionkowski, Mon. Not. R. Astron. Soc. 313, 141 (2000).

[24] E. Komatsu and D. N. Spergel, Phys. Rev. D63, 063002 (2001).

[25] M. Sasaki, J. Väliviita, and D. Wands, Phys. Rev. D 74, 103003 (2006).

[26] K. Enqvist and T. Takahashi, J. Cosmol. Astropart. Phys. 92008, 012

[27] Q.-G. Huang and Y. Wang, J. Cosmol. Astropart. Phys. 92008, 025.

[28] Q.-G. Huang, J. Cosmol. Astropart. Phys. 62009, 035.

[29] C. T. Byrnes and G. Tasinato, J. Cosmol. Astropart. Phys. 8 (2009) 016.

[30] P.D. Meerburg, J.P. van der Schaar, and P. Stefano Corasaniti, J. Cosmol. Astropart. Phys. 52009, 018.

[31] L. Verde and S. Matarrese, Astrophys. J. Lett. 706, L91 (2009).

[32] L. Senatore, K. M. Smith, and M. Zaldarriaga, J. Cosmol. Astropart. Phys. 1 (2010) 028.

[33] Even though this shape does not correspond to any physical mechanism generating primordial NG, we will consider it since it has been simulated [15].

[34] P. Creminelli, A. Nicolis, L. Senatore, M. Tegmark, and M. Zaldarriaga, J. Cosmol. Astropart. Phys. 52006, 004.

[35] N. Kaiser, Astrophys. J. Lett. 284, L9 (1984).

[36] E. Komatsu, et al., Astrophys. J. Suppl. Ser. 192, 18 (2011).

[37] W.H. Press and P. Schechter, Astrophys. J. 187, 425 (1974).
[38] J. E. Gunn and J. R. Gott, III, Astrophys. J. 176, 1 (1972).

[39] A complete derivation can be found in Appendix $\mathrm{K}$ of [40].

[40] D. Jeong, Ph. D. thesis, University of Texas at Austin, 2010.

[41] L. G. Jensen and A. S. Szalay, Astrophys. J. Lett. 305, L5 (1986).

[42] J. N. Fry and E. Gaztanaga, Astrophys. J. 413, 447 (1993).

[43] T. Matsubara, Phys. Rev. D83, 083518 (2011).

[44] A. Taruya, K. Koyama, and T. Matsubara, Phys. Rev. D 78, 123534 (2008).

[45] E. Sefusatti, Phys. Rev. D80, 123002 (2009).

[46] D. Jeong and E. Komatsu, Astrophys. J. 703, 1230 (2009).

[47] P. McDonald, Phys. Rev. D 78, 123519 (2008).

[48] R. K. Sheth and G. Tormen, Mon. Not. R. Astron. Soc. 308, 119 (1999).

[49] R. K. Sheth and G. Tormen, Mon. Not. R. Astron. Soc. 329, 61 (2002).

[50] T. Giannantonio and C. Porciani, Phys. Rev. D 81, 063530 (2010).

[51] This is true excluding unviable kernels that yield large loop corrections to the power spectrum $P_{\phi}$.

[52] S. Matarrese, L. Verde, and R. Jimenez, Astrophys. J. 541, 10 (2000).

[53] M. Lo Verde, A. Miller, S. Shandera, and L. Verde, J. Cosmol. Astropart. Phys. 4 (2008) 014.

[54] T. Y. Lam and R. K. Sheth, Mon. Not. R. Astron. Soc. 398, 2143 (2009).

[55] M. Maggiore and A. Riotto, Mon. Not. R. Astron. Soc. 405, 1244 (2010).

[56] P. Valageas, Astron. Astrophys. 514, A46 (2010).

[57] G. D’Amico, M. Musso, J. Noreña, and A. Paranjape, arXiv:1005.1203.

[58] M. LoVerde and K. M. Smith, J. Cosmol. Astropart. Phys. 8 (2011) 003.

[59] S. Yokoyama, N. Sugiyama, S. Zaroubi, and J. Silk, arXiv:1103.2586.

[60] S. Chongchitnan and J. Silk, Astrophys. J. 724, 285 (2010).

[61] K. Enqvist, S. Hotchkiss, and O. Taanila, J. Cosmol. Astropart. Phys. 4 (2011) 017.

[62] T. Matsubara, Astrophys. J. 584, 1 (2003).

[63] S. Chongchitnan and J. Silk, Phys. Rev. D 83, 083504 (2011).

[64] A. Slosar, J. Cosmol. Astropart. Phys. 32009, 004.

[65] U. Seljak, N. Hamaus, and V. Desjacques, Phys. Rev. Lett. 103, 091303 (2009).

[66] N. Hamaus, U. Seljak, V. Desjacques, R. E. Smith, and T. Baldauf, Phys. Rev. D 82, 043515 (2010).

[67] Y. Cai, G. Bernstein, and R. K. Sheth, Mon. Not. R. Astron. Soc. 412995 (2011).

[68] N. Hamaus, U. Seljak, and V. Desjacques, arXiv:1104.2321.

[69] G. M. Bernstein and Y.-C. Cai, arXiv:1104.3862.

[70] M. Maggiore and A. Riotto, Astrophys. J. 717, 526 (2010).

[71] A. De Simone, M. Maggiore, and A. Riotto, Mon. Not. R. Astron. Soc., 4122587 (2011). 